\title{
Dark Energy Survey year 1 results: Cosmological constraints from galaxy clustering and weak lensing
}

T. M. C. Abbott, ${ }^{1}$ F. B. Abdalla, ${ }^{2,3}$ A. Alarcon, ${ }^{4}$ J. Aleksić, ${ }^{5}$ S. Allam, ${ }^{6}$ S. Allen, ${ }^{7}$ A. Amara, ${ }^{8}$ J. Annis, ${ }^{6}$ J. Asorey, ${ }^{9,10}$ S. Avila, ${ }^{11,12}$ D. Bacon,${ }^{11}$ E. Balbinot,${ }^{13}$ M. Banerii ${ }^{14,15}$ N. Banik,${ }^{6}$ W. Barkhouse, ${ }^{16}$ M. Baumer, ${ }^{7,17,18}$ E. Baxter ${ }^{19}$ K. Bechtol, ${ }^{20}$ M. R. Becker, ${ }^{7,17}$ A. Benoit-Lévy, ${ }^{3,21,22}$ B. A. Benson, ${ }^{6,23}$ G. M. Bernstein, ${ }^{19}$ E. Bertin, ${ }^{22,21}$ J. Blazek, ${ }^{24,25}$ S. L. Bridle, ${ }^{26}$ D. Brooks, ${ }^{3}$ D. Brout,${ }^{19}$ E. Buckley-Geer, ${ }^{6}$ D. L. Burke, ${ }^{17,18}$ M. T. Busha, ${ }^{17}$ A. Campos, ${ }^{27,28}$ D. Capozzi, ${ }^{11}$ A. Carnero Rosell, ${ }^{28,29}$ M. Carrasco Kind ${ }^{30,31}$ J. Carretero, ${ }^{5}$ F. J. Castander, ${ }^{4}$ R. Cawthon, ${ }^{23}$ C. Chang, ${ }^{23}$ N. Chen, ${ }^{23}$ M. Childress, ${ }^{32}$ A. Choi, ${ }^{25}$ C. Conselice,${ }^{33}$ R. Crittenden, ${ }^{11}$ M. Crocce, ${ }^{4}$ C. E. Cunha, ${ }^{17}$ C. B. D'Andrea, ${ }^{19}$ L. N. da Costa, ${ }^{28,29}$ R. Das ${ }^{34}$ T. M. Davis, ${ }^{9,10}$ C. Davis, ${ }^{17}$ J. De Vicente, ${ }^{35}$ D. L. DePoy, ${ }^{36}$ J. DeRose, ${ }^{7,17}$ S. Desai, ${ }^{37}$ H. T. Diehl, ${ }^{6}$ J. P. Dietrich, ${ }^{38,39}$ S. Dodelson, ${ }^{6,23}$ P. Doel, ${ }^{3}$ A. Drlica-Wagner, ${ }^{6}$ T. F. Eifler, ${ }^{40,41}$ A. E. Elliott, ${ }^{42}$ F. Elsner, ${ }^{3}$ J. Elvin-Poole ${ }^{26}$ J. Estrada, ${ }^{6}$ A. E. Evrard, ${ }^{43,34}$ Y. Fang, ${ }^{19}$ E. Fernandez, ${ }^{5}$ A. Ferté, ${ }^{44}$ D. A. Finley, ${ }^{6}$ B. Flaugher, ${ }^{6}$ P. Fosalba, ${ }^{4}$ O. Friedrich,${ }^{45,46}$ J. Frieman, ${ }^{23,6}$ J. García-Bellido, ${ }_{18}$ M. Garcia-Fernandez, ${ }^{35}$ M. Gatti, ${ }^{5}$ E. Gaztanaga, ${ }^{4}$ D. W. Gerdes, ${ }^{34,43}$

T. Giannantonio, ${ }^{45,15,14}$ M. S. S. Gill, ${ }^{18}$ K. Glazebrook, ${ }^{47}$ D. A. Goldstein, ${ }^{48,49}$ D. Gruen,${ }^{50,17,18}$ R. A. Gruendl, ${ }^{31,30}$ J. Gschwend, ${ }^{28,29}$ G. Gutierrez, ${ }^{6}$ S. Hamilton, ${ }^{34}$ W. G. Hartley, ${ }^{3,8}$ S. R. Hinton, ${ }^{9}$ K. Honscheid ${ }^{25,42}$ B. Hoyle, ${ }^{45}$ D. Huterer, ${ }^{34}$ B. Jain, ${ }^{19}$ D. J. James ${ }^{51}$ M. Jarvis ${ }^{19}{ }^{19}$ T. Jeltema ${ }^{52}$ M. D. Johnson, ${ }^{30}$ M. W. G. Johnson ${ }^{30}$ T. Kacprzak, ${ }^{8}$ S. Kent, ${ }^{23,6}$ A. G. Kim, ${ }^{49}$ A. King, ${ }^{9}$ D. Kirk, ${ }^{3}$ N. Kokron, ${ }^{53}$ A. Kovacs, ${ }^{5}$ E. Krause, ${ }^{17}$ C. Krawiec, ${ }^{19}$ A. Kremin, ${ }^{34}$ K. Kuehn, ${ }^{54}$ S. Kuhlmann, ${ }^{55}$ N. Kuropatkin, ${ }^{6}$ F. Lacasa, ${ }^{27}$ O. Lahav, ${ }^{3}$ T. S. Li, ${ }^{6}$ A. R. Liddle, ${ }^{44}$ C. Lidman, ${ }^{10,54}$ M. Lima ${ }^{28,53}$ H. Lin,${ }^{6}$ N. MacCrann, ${ }^{42,25}$ M. A. G. Maia, ${ }^{29,28}$ M. Makler, ${ }^{56}$ M. Manera, ${ }^{3}$ M. March, ${ }^{19}$ J. L. Marshall, ${ }^{36}$ P. Martini, ${ }^{57,25}$ R. G. McMahon, ${ }^{14,15}$ P. Melchior, ${ }^{58}$ F. Menanteau, ${ }^{30,31}$ R. Miquel, ${ }^{5,59}$ V. Miranda, ${ }^{19}$ D. Mudd, ${ }^{57}$ J. Muir, ${ }^{34}$ A. Möller, ${ }^{60,10}$ E. Neilsen, ${ }^{6}$ R. C. Nichol, ${ }_{11}^{11}$ B. Nord, ${ }^{6}$ P. Nugent, ${ }^{49}$ R. L. C. Ogando,${ }^{29,28}$ A. Palmese, ${ }^{3}$ J. Peacock, ${ }^{44}$ H. V. Peiris, ${ }^{3}$ J. Peoples, ${ }^{6}$ W. J. Percival, ${ }^{11}$ D. Petravick, ${ }^{30}$ A. A. Plazas, ${ }^{41}$ A. Porredon, ${ }^{4}$ J. Prat, ${ }^{5}$ A. Pujol, ${ }^{4}$ M. M. Rau, ${ }^{45}$ A. Refregier, ${ }^{8}$ P. M. Ricker ${ }^{31,30}$ N. Roe, ${ }^{49}$ R. P. Rollins, ${ }^{26}$ A. K. Romer ${ }^{61}$ A. Roodman, ${ }^{17,18}$ R. Rosenfeld, ${ }^{27,28}$ A. J. Ross, ${ }^{25}$ E. Rozo, ${ }^{62}$ E. S. Rykoff, ${ }^{17,18}$ M. Sako, ${ }^{19}$ A. I. Salvador ${ }^{35}$ S. Samuroff, ${ }^{26}$ C. Sánchez, ${ }^{5}$ E. Sanchez,${ }^{35}$ B. Santiago,${ }^{63,28}$ V. Scarpine, ${ }^{6}$ R. Schindler, ${ }^{18}$ D. Scolnic, ${ }^{23}$ L. F. Secco, ${ }^{19}$ S. Serrano, ${ }^{4}$ I. Sevilla-Noarbe,${ }^{35}$ E. Sheldon, ${ }^{64}$ R. C. Smith, ${ }^{1}$ M. Smith, ${ }^{32}$ J. Smith ${ }^{65}$ M. Soares-Santos, ${ }^{6}$ F. Sobreira, ${ }^{28,66}$ E. Suchyta, ${ }^{67}$ G. Tarle, ${ }^{34}$ D. Thomas, ${ }^{11}$ M. A. Troxel, ${ }^{42,25}$ D. L. Tucker, ${ }^{6}$ B. E. Tucker, ${ }^{10,60}$ S. A. Uddin, ${ }^{10,68}$ T. N. Varga, ${ }^{46,45}$ P. Vielzeuf, ${ }^{5}$ V. Vikram, ${ }^{55}$ A. K. Vivas, ${ }^{1}$ A. R. Walker, ${ }^{1}$ M. Wang, ${ }^{6}$ R. H. Wechsler, ${ }^{18,17,7}$ J. Weller, ${ }^{38,45,46}$ W. Wester, ${ }^{6}$ R. C. Wolf, ${ }^{19}$ B. Yanny, ${ }^{6}$ F. Yuan, ${ }^{10,60}$ A. Zenteno, ${ }^{1}$

B. Zhang, ${ }^{60,10}$ Y. Zhang, ${ }^{6}$ and J. Zuntz ${ }^{44}$

\section{(Dark Energy Survey Collaboration) ${ }^{*}$}

${ }^{1}$ Cerro Tololo Inter-American Observatory, National Optical Astronomy Observatory, Casilla 603, La Serena, Chile

${ }^{2}$ Department of Physics and Electronics, Rhodes University, P.O. Box 94, Grahamstown 6140, South Africa

${ }^{3}$ Department of Physics \& Astronomy, University College London, Gower Street, London WCIE 6BT, United Kingdom

${ }^{4}$ Institute of Space Sciences, IEEC-CSIC, Campus UAB, Carrer de Can Magrans, $s / n, 08193$ Barcelona, Spain

${ }^{5}$ Institut de Física d'Altes Energies (IFAE), The Barcelona Institute of Science and Technology, Campus UAB, 08193 Bellaterra (Barcelona), Spain

${ }^{6}$ Fermi National Accelerator Laboratory, P.O. Box 500, Batavia, Illinois 60510, USA ${ }^{7}$ Department of Physics, Stanford University, 382 Via Pueblo Mall, Stanford, California 94305, USA

${ }^{8}$ Department of Physics, ETH Zurich, Wolfgang-Pauli-Strasse 16, CH-8093 Zurich, Switzerland

${ }^{9}$ School of Mathematics and Physics, University of Queensland, Brisbane QLD 4072, Australia

${ }^{10}$ ARC Centre of Excellence for All-sky Astrophysics (CAASTRO)

${ }^{11}$ Institute of Cosmology \& Gravitation, University of Portsmouth, Portsmouth PO1 3FX, United Kingdom

${ }^{12}$ Instituto de Fisica Teorica UAM/CSIC, Universidad Autonoma de Madrid, 28049 Madrid, Spain

${ }^{13}$ Department of Physics, University of Surrey, Guildford GU2 7XH, United Kingdom

${ }^{14}$ Institute of Astronomy, University of Cambridge,

Madingley Road, Cambridge CB3 OHA, United Kingdom

${ }^{15}$ Kavli Institute for Cosmology, University of Cambridge,

Madingley Road, Cambridge CB3 OHA, United Kingdom 
${ }^{16}$ University of North Dakota, Department of Physics and Astrophysics,

Witmer Hall, Grand Forks, North Dakota 58202, USA

${ }^{17}$ Kavli Institute for Particle Astrophysics \& Cosmology,

P.O. Box 2450, Stanford University, Stanford, California 94305, USA

${ }^{18}$ SLAC National Accelerator Laboratory, Menlo Park, California 94025, USA

${ }^{19}$ Department of Physics and Astronomy, University of Pennsylvania,

Philadelphia, Pennsylvania 19104, USA

${ }^{20}$ LSST, 933 North Cherry Avenue, Tucson, Arizona 85721, USA

${ }^{21}$ CNRS, UMR 7095, Institut d'Astrophysique de Paris, F-75014 Paris, France

${ }^{22}$ Sorbonne Universités, UPMC Univ Paris 06, UMR 7095,

Institut d'Astrophysique de Paris, F-75014 Paris, France

${ }^{23}$ Kavli Institute for Cosmological Physics, University of Chicago, Chicago, Illinois 60637, USA

${ }^{24}$ Institute of Physics, Laboratory of Astrophysics, École Polytechnique Fédérale de Lausanne (EPFL), Observatoire de Sauverny, 1290 Versoix, Switzerland

${ }^{25}$ Center for Cosmology and Astro-Particle Physics, The Ohio State University, Columbus, Ohio 43210, USA

${ }^{26}$ Jodrell Bank Center for Astrophysics, School of Physics and Astronomy, University of Manchester, Oxford Road, Manchester M13 9PL, United Kingdom

${ }^{27}$ ICTP South American Institute for Fundamental Research Instituto de Física Teórica, Universidade Estadual Paulista, São Paulo, Brazil

${ }^{28}$ Laboratório Interinstitucional de e-Astronomia-LIneA,

Rua Gal. José Cristino 77, Rio de Janeiro, Rio de Janeiro 20921-400, Brazil

${ }^{29}$ Observatório Nacional, Rua Gal. José Cristino 77, Rio de Janeiro, Rio de Janeiro 20921-400, Brazil

${ }^{30}$ National Center for Supercomputing Applications, 1205 West Clark Street, Urbana, Illinois 61801, USA

${ }^{31}$ Department of Astronomy, University of Illinois, 1002 West Green Street, Urbana, Illinois 61801, USA

${ }^{32}$ School of Physics and Astronomy, University of Southampton, Southampton SO17 1BJ, United Kingdom

${ }^{33}$ University of Nottingham, School of Physics and Astronomy, Nottingham NG7 2RD, United Kingdom

${ }^{34}$ Department of Physics, University of Michigan, Ann Arbor, Michigan 48109, USA

${ }^{35}$ Centro de Investigaciones Energéticas, Medioambientales y Tecnológicas (CIEMAT), Madrid, Spain

${ }^{36}$ George P. and Cynthia Woods Mitchell Institute for Fundamental Physics and Astronomy, and

Department of Physics and Astronomy, Texas A\&M University, College Station, Texas 77843, USA

${ }^{37}$ Department of Physics, IIT Hyderabad, Kandi, Telangana 502285, India

${ }^{38}$ Excellence Cluster Universe, Boltzmannstr. 2, 85748 Garching, Germany

${ }^{39}$ Faculty of Physics, Ludwig-Maximilians-Universität, Scheinerstr. 1, 81679 Munich, Germany

${ }^{40}$ Department of Physics, California Institute of Technology, Pasadena, California 91125, USA

${ }^{41}$ Jet Propulsion Laboratory, California Institute of Technology, 4800 Oak Grove Drive, Pasadena, California 91109, USA

${ }^{42}$ Department of Physics, The Ohio State University, Columbus, Ohio 43210, USA

${ }^{43}$ Department of Astronomy, University of Michigan, Ann Arbor, Michigan 48109, USA

${ }^{44}$ Institute for Astronomy, University of Edinburgh, Edinburgh EH9 3HJ, United Kingdom

${ }^{45}$ Universitäts-Sternwarte, Fakultät für Physik, Ludwig-Maximilians Universität München, Scheinerstr. 1, 81679 München, Germany

${ }^{46}$ Max Planck Institute for Extraterrestrial Physics, Giessenbachstrasse, 85748 Garching, Germany

${ }^{47}$ Centre for Astrophysics \& Supercomputing, Swinburne University of Technology, Victoria 3122, Australia

${ }^{48}$ Department of Astronomy, University of California, Berkeley, 501 Campbell Hall, Berkeley, California 94720, USA

${ }^{49}$ Lawrence Berkeley National Laboratory, 1 Cyclotron Road, Berkeley, California 94720, USA

${ }^{50}$ Einstein Fellow

${ }^{51}$ Astronomy Department, University of Washington, Box 351580, Seattle, Washington 98195, USA

${ }^{52}$ Santa Cruz Institute for Particle Physics, Santa Cruz, California 95064, USA

${ }^{53}$ Departamento de Física Matemática, Instituto de Física, Universidade de São Paulo, CP 66318, São Paulo, São Paulo 05314-970, Brazil

${ }^{54}$ Australian Astronomical Observatory, North Ryde, New South Wales 2113, Australia

${ }^{55}$ Argonne National Laboratory, 9700 South Cass Avenue, Lemont, Illinois 60439, USA

${ }^{56}$ ICRA, Centro Brasileiro de Pesquisas Físicas, Rua Dr. Xavier Sigaud 150, CEP 22290-180, Rio de Janeiro, Rio de Janeiro, Brazil

${ }^{57}$ Department of Astronomy, The Ohio State University, Columbus, Ohio 43210, USA

${ }^{58}$ Department of Astrophysical Sciences, Princeton University, Peyton Hall, Princeton, New Jersey 08544, USA 


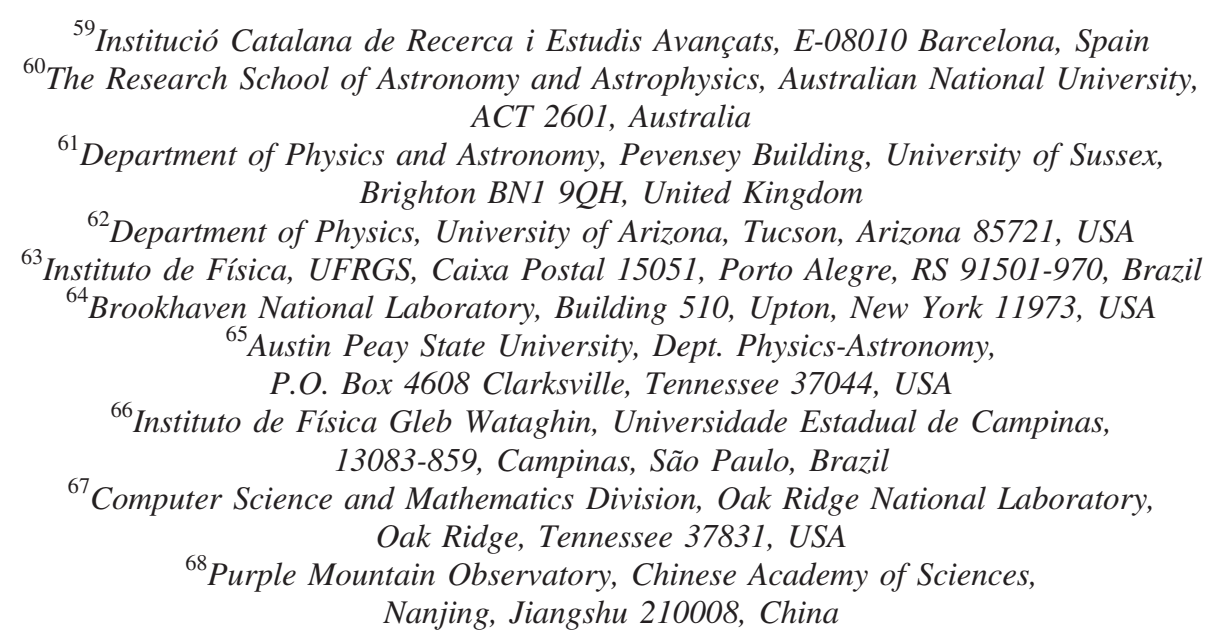

(Received 10 August 2017; published 27 August 2018)

\begin{abstract}
We present cosmological results from a combined analysis of galaxy clustering and weak gravitational lensing, using $1321 \mathrm{deg}^{2}$ of griz imaging data from the first year of the Dark Energy Survey (DES Y1). We combine three two-point functions: (i) the cosmic shear correlation function of 26 million source galaxies in four redshift bins, (ii) the galaxy angular autocorrelation function of 650,000 luminous red galaxies in five redshift bins, and (iii) the galaxy-shear cross-correlation of luminous red galaxy positions and source galaxy shears. To demonstrate the robustness of these results, we use independent pairs of galaxy shape, photometric-redshift estimation and validation, and likelihood analysis pipelines. To prevent confirmation bias, the bulk of the analysis was carried out while "blind" to the true results; we describe an extensive suite of systematics checks performed and passed during this blinded phase. The data are modeled in flat $\Lambda \mathrm{CDM}$ and $w C D M$ cosmologies, marginalizing over 20 nuisance parameters, varying 6 (for $\Lambda \mathrm{CDM}$ ) or 7 (for $w \mathrm{CDM})$ cosmological parameters including the neutrino mass density and including the $457 \times 457$ element analytic covariance matrix. We find consistent cosmological results from these three two-point functions and from their combination obtain $S_{8} \equiv \sigma_{8}\left(\Omega_{m} / 0.3\right)^{0.5}=0.773_{-0.020}^{+0.026}$ and $\Omega_{m}=0.267_{-0.017}^{+0.030}$ for $\Lambda \mathrm{CDM}$; for $w \mathrm{CDM}$, we find $S_{8}=0.782_{-0.024}^{+0.036}, \Omega_{m}=0.284_{-0.030}^{+0.033}$, and $w=-0.82_{-0.20}^{+0.21}$ at $68 \%$ C.L. The precision of these DES Y1 constraints rivals that from the Planck cosmic microwave background measurements, allowing a comparison of structure in the very early and late Universe on equal terms. Although the DES Y1 best-fit values for $S_{8}$ and $\Omega_{m}$ are lower than the central values from Planck for both $\Lambda$ CDM and $w$ CDM, the Bayes factor indicates that the DES Y1 and Planck data sets are consistent with each other in the context of $\Lambda$ CDM. Combining DES Y1 with Planck, baryonic acoustic oscillation measurements from SDSS, 6dF, and BOSS and type Ia supernovae from the Joint Lightcurve Analysis data set, we derive very tight constraints on cosmological parameters: $S_{8}=0.802 \pm 0.012$ and $\Omega_{m}=0.298 \pm 0.007$ in $\Lambda \mathrm{CDM}$ and $w=-1.00_{-0.04}^{+0.05}$ in $w C D M$. Upcoming Dark Energy Survey analyses will provide more stringent tests of the $\Lambda \mathrm{CDM}$ model and extensions such as a time-varying equation of state of dark energy or modified gravity.
\end{abstract}

DOI: $10.1103 /$ PhysRevD.98.043526

\section{INTRODUCTION}

The discovery of cosmic acceleration [1,2] established the cosmological constant $(\Lambda)$ [3] + cold dark matter $(\Lambda \mathrm{CDM})$ model as the standard cosmological paradigm that explains a wide variety of phenomena, from the origin and evolution of large-scale structure to the current epoch of accelerated expansion [4,5]. The successes of $\Lambda$ CDM, however, must be balanced by its apparent implausibility:

*For correspondence use des-publication-queries@fnal.gov three new entities beyond the Standard Model of particle physics-one that drove an early epoch of inflation, another that serves as dark matter, and a third that is driving the current epoch of acceleration-are required, none of them easily connected to the rest of physics [6]. Ongoing and planned cosmic surveys are designed to test $\Lambda \mathrm{CDM}$ and more generally to shed light on the mechanism driving the current epoch of acceleration, be it the vacuum energy associated with the cosmological constant, another form of dark energy, a modification of General Relativity, or something more drastic. 
The Dark Energy Survey (DES) ${ }^{1}$ [7] is an ongoing, fiveyear survey that, when completed, will map 300 million galaxies and tens of thousands of galaxy clusters in five filters ( griz $Y$ ) over $5000 \mathrm{deg}^{2}$, in addition to discovering several thousand type Ia supernovae in a $27 \mathrm{deg}^{2}$ timedomain survey. DES will use several cosmological probes to test $\Lambda \mathrm{CDM}$; galaxy clustering and weak gravitational lensing are two of the most powerful. Jointly, these complementary probes sample the underlying matter density field through the galaxy population and the distortion of light due to gravitational lensing. In this paper, we use data on this combination from the first year (Y1) of DES to constrain $\Lambda \mathrm{CDM}$ and its simplest extension- $w \mathrm{CDM}$, having a free parameter for the dark energy equation of state.

The spatial distribution of galaxies in the Universe, and its temporal evolution, carry important information about the physics of the early Universe as well as details of structure evolution in the late Universe, thereby testing some of the most precise predictions of $\Lambda \mathrm{CDM}$. Indeed, measurements of the galaxy two-point correlation function, the lowest-order statistic describing the galaxy spatial distribution, provided early evidence for the $\Lambda$ CDM model [8-19]. The data-model comparison in this case depends upon uncertainty in the galaxy bias [20], the relation between the galaxy spatial distribution and the theoretically predicted matter distribution.

In addition to galaxy clustering, weak gravitational lensing has become one of the principal probes of cosmology. While the interpretation of galaxy clustering is complicated by galaxy bias, weak lensing provides direct measurement of the mass distribution via cosmic shear, the correlation of the apparent shapes of pairs of galaxies induced by foreground large-scale structure. Further information on the galaxy bias is provided by galaxy-galaxy lensing, the cross-correlation of lens galaxy positions and source galaxy shapes.

The shape distortions produced by gravitational lensing, while cosmologically informative, are extremely difficult to measure, since the induced source galaxy ellipticities are at the percent level, and a number of systematic effects can obscure the signal. Indeed, the first detections of weak lensing were made by cross-correlating observed shapes of source galaxies with massive foreground lenses [21,22]. A watershed moment came in the year 2000 when four research groups nearly simultaneously announced the first detections of cosmic shear [23-26]. While these and subsequent weak lensing measurements are also consistent with $\Lambda \mathrm{CDM}$, only recently have they begun to provide competitive constraints on cosmological parameters [27-36]. Galaxy-galaxy lensing measurements have also matured to the point where their combination with galaxy clustering breaks degeneracies between the cosmological

\footnotetext{
${ }^{1}$ http://www.darkenergysurvey.org/.
}

parameters and bias, thereby helping to constrain dark energy [22,37-48]. The combination of galaxy clustering, cosmic shear, and galaxy-galaxy lensing measurements powerfully constrains structure formation in the late Universe. As for cosmological analyses of samples of galaxy clusters (see Ref. [49] for a review), redshift space distortions in the clustering of galaxies (see Ref. [50] and references therein), and other measurements of late-time structure, a primary test is whether these are consistent, in the framework of $\Lambda \mathrm{CDM}$, with measurements from cosmic microwave background (CMB) experiments that are chiefly sensitive to early-Universe physics [51-54] as well as lensing of its photons by the large-scale structures (e.g., Refs. [55-57]).

The main purpose of this paper is to combine the information from galaxy clustering and weak lensing, using the galaxy and shear correlation functions as well as the galaxy-shear cross-correlation. It has been recognized for more than a decade that such a combination contains a tremendous amount of complementary information, as it is remarkably resilient to the presence of nuisance parameters that describe systematic errors and noncosmological information [58-61]. It is perhaps simplest to see that the combined analysis could separately solve for galaxy bias and the cosmological parameters; however, it can also internally solve for (or self-calibrate [62]) the systematics associated with photometric redshifts [63-65], intrinsic alignment [66], and a wide variety of other effects [60]. Such a combined analysis has recently been executed by combining the KiDS $450 \mathrm{deg}^{2}$ weak lensing survey with two different spectroscopic galaxy surveys $[67,68]$. While these multiprobe analyses still rely heavily on prior information about the nuisance parameters, obtained through a wide variety of physical tests and simulations, this approach does significantly mitigate potential biases due to systematic errors and will likely become even more important as statistical errors continue to drop. The multiprobe analyses also extract more precise information about cosmology from the data than any single measurement could.

Previously, the DES Collaboration analyzed data from the Science Verification period, which covered $139 \mathrm{deg}^{2}$, carrying out several path-finding analyses of galaxy clustering and gravitational lensing, along with numerous others $[46,48,69-83]$. The DES Y1 data set analyzed here covers about ten times more area, albeit shallower, and provides 650,000 lens galaxies and the shapes of 26 million source galaxies, each of them divided into redshift bins. The lens sample comprises bright, red-sequence galaxies, which have secure photometric-redshift (photo- $z$ ) estimates. We measure three two-point functions from these data: (i) $w(\theta)$, the angular correlation function of the lens galaxies; (ii) $\gamma_{t}(\theta)$, the correlation of the tangential shear of sources with lens galaxy positions; and (iii) $\xi_{ \pm}(\theta)$, the correlation functions of different components of the 
ellipticities of the source galaxies. We use these measurements only on large angular scales, for which we have verified that a relatively simple model describes the data, although, even with this restriction, we must introduce 20 parameters to capture astrophysical and measurementrelated systematic uncertainties.

This paper is built upon, and uses tools and results from, 11 other papers:

(i) Ref. [84] describes the theory and parameter-fitting methodologies, including the binning and modeling of all the two-point functions, the marginalization of astrophysical and measurement-related uncertainties, and the ways in which we calculate the covariance matrix and obtain the ensuing parameter constraints;

(ii) Ref. [85], which applies this methodology to image simulations generated to mimic many aspects of the Y1 data sets;

(iii) a description of the process by which the valueadded galaxy catalog (Y1 Gold) is created from the data and the tests on it to ensure its robustness [86];

(iv) a shape catalog paper, which presents the two shape catalogs generated using two independent techniques and the many tests carried out to ensure that residual systematic errors in the inferred shear estimates are sufficiently small for $\mathrm{Y} 1$ analyses Refs. [87];

(v) Ref. [88], which describes how the redshift distributions of galaxies in these shape catalogs are estimated from their photometry, including a validation of these estimates by means of COSMOS multiband photometry;

(vi) three papers [89-91] that describe the use of angular cross-correlation with samples of secure redshifts to independently validate the photometric-redshift distributions of lens and source galaxies;

(vii) Ref. [92], which measures and derives cosmological constraints from the cosmic shear signal in the DES Y1 data and also addresses the question of whether DES lensing data are consistent with lensing results from other surveys;

(viii) Ref. [93], which describes galaxy-galaxy lensing results, including a wide variety of tests for systematic contamination and a cross-check on the redshift distributions of source galaxies using the scaling of the lensing signal with redshift;

(ix) Ref. [94], which describes the galaxy clustering statistics, including a series of tests for systematic contamination-this paper also describes updates to the REDMAGIC algorithm used to select our lens galaxies and to estimate their photometric redshifts.

Armed with the above results, this paper presents the most stringent cosmological constraints from a galaxy imaging survey to date and, combined with external data, the most stringent constraints overall.
One of the guiding principles of the methods developed in these papers is redundancy: we use two independent shape measurement methods that are independently calibrated, several photometric-redshift estimation and validation techniques, and two independent codes for predicting our signals and performing a likelihood analysis. Comparison of these, as described in the above papers, has been an important part of the verification of each step of our analysis.

The plan of the paper is as follows. Section II gives an overview of the data used in the analysis, while Sec. III presents the two-point statistics that contain the relevant information about cosmological parameters. Section IV describes the methodology used to compare these statistics to theory, thereby extracting cosmological results. We validated our methodology while remaining blinded to the results of the analyses; this process is described in Sec. V, and some of the tests that convinced us to unblind are recounted in Appendix A. Section VI presents the cosmological results from these three probes as measured by DES in the context of two models, $\Lambda \mathrm{CDM}$ and $w \mathrm{CDM}$, while Sec. VII compares DES results with those from other experiments, offering one of the most powerful tests to date of $\Lambda \mathrm{CDM}$. Then, we combine DES with external data sets with which it is consistent to produce the tightest constraints yet on cosmological parameters. Finally, we conclude in Sec. VIII. Appendix B presents further evidence of the robustness of our results. And Appendix C describes updates in the covariance matrix calculation carried out after the first version of this paper had been posted.

\section{DATA}

DES uses the 570-megapixel Dark Energy Camera (DECam) [95], built by the collaboration and deployed on the Cerro Tololo Inter-American Observatory $4 \mathrm{~m}$ Blanco telescope in Chile, to image the South Galactic Cap in the griz $Y$ filters. In this paper, we analyze DECam images taken from August 31, 2013, to February 9, 2014 ("DES Year 1" or Y1), covering $1786 \mathrm{deg}^{2}$ in griz after coaddition and before masking [86]. The data were processed through the DES Data Management (DESDM) system [96-99], which detrends and calibrates the raw DES images, combines individual exposures to create coadded images, and detects and catalogs astrophysical objects. Further vetting and subselection of the DESDM data products was performed by [86] to produce a high-quality object catalog (Y1 Gold) augmented by several ancillary data products including a star/galaxy separator. With up to four exposures per filter per field in Y1, and individual griz exposures of $90 \mathrm{sec}$ and $Y$ exposures of $45 \mathrm{sec}$, the characteristic $10 \sigma$ limiting magnitude for galaxies is $g=23.4, \quad r=23.2, \quad i=22.5, \quad z=21.8$, and $Y=20.1$ [86]. Additional analyses produced catalogs of red galaxies, photometric-redshift estimates, and galaxy shape estimates, as described below. 
As noted in the Introduction, we use two samples of galaxies in the current analysis: lens galaxies, for the angular clustering measurement, and source galaxies, the shapes of which we estimate and correlate with each other ("cosmic shear"). The tangential shear is measured for the source galaxies about the positions of the lens galaxies (galaxy-galaxy lensing).

\section{A. Lens galaxies}

We rely on REDMAGIC galaxies for all galaxy clustering measurements [94] and as the lens population for the galaxy-galaxy lensing analysis [93]. They have the advantage of being easily identifiable, being relatively strongly clustered, and having relatively small photometric-redshift errors; they are selected using a simple algorithm [100]:

(1) Fit every galaxy in the survey to a red-sequence template, and compute the corresponding best-fit redshift $z_{\text {red }}$.

(2) Evaluate the goodness of fit $\chi^{2}$ of the red-sequence template and the galaxy luminosity, using the assigned photometric redshift.

(3) Include the galaxy in the REDMAGIC catalog if and only if it is bright $\left(L \geq L_{\min }\right)$ and the red-sequence template is a good fit $\left(\chi^{2} \leq \chi_{\max }^{2}\right)$.

In practice, we do not specify $\chi_{\max }^{2}$ but instead demand that the resulting galaxy sample have a constant comoving density as a function of redshift. Consequently, REDMAGIC galaxy selection depends upon only two parameters: the selected luminosity threshold, $L_{\min }$, and the comoving density, $\bar{n}$, of the sample. Of course, not all combinations of parameters are possible; brighter galaxy samples must be less dense.

Three separate REDMAGIC samples were generated from the Y1 data, referred to as the high-density, high-luminosity, and higher-luminosity samples. The corresponding luminosity thresholds ${ }^{2}$ and comoving densities for these samples are, respectively, $L_{\min }=0.5 L_{*}, L_{*}$, and $1.5 L_{*}$ and $\bar{n}=10^{-3}, 4 \times 10^{-4}$, and $10^{-4}$ galaxies $/\left(h^{-1} \mathrm{Mpc}\right)^{3}$, where $h \equiv H_{0} /\left(100 \mathrm{~km} \mathrm{sec}^{-1} \mathrm{Mpc}^{-1}\right)$ parametrizes the Hubble constant. Naturally, brighter galaxies are easier to map at higher redshifts than the dimmer galaxies are. These galaxies are placed in five nominally disjoint redshift bins. The lowest three bins $z=[(0.15-0.3),(0.3-$ $0.45),(0.45-0.6)]$ are high density, while the galaxies in the two highest redshift bins $((0.6-0.75)$ and $(0.75-0.9))$ are high luminosity and higher luminosity, respectively. The estimated redshift distributions of these five binned lens galaxy samples are shown in the upper panel of Fig. 1.

The clustering properties of these galaxies are an essential part of this combined analysis, so great care is taken in Ref. [94] to ensure that the galaxy maps are not

\footnotetext{
${ }^{2}$ Here and throughout, whenever a cosmology is required, we use $\Lambda C D M$ with the parameters given in Table 1 of [84].
}

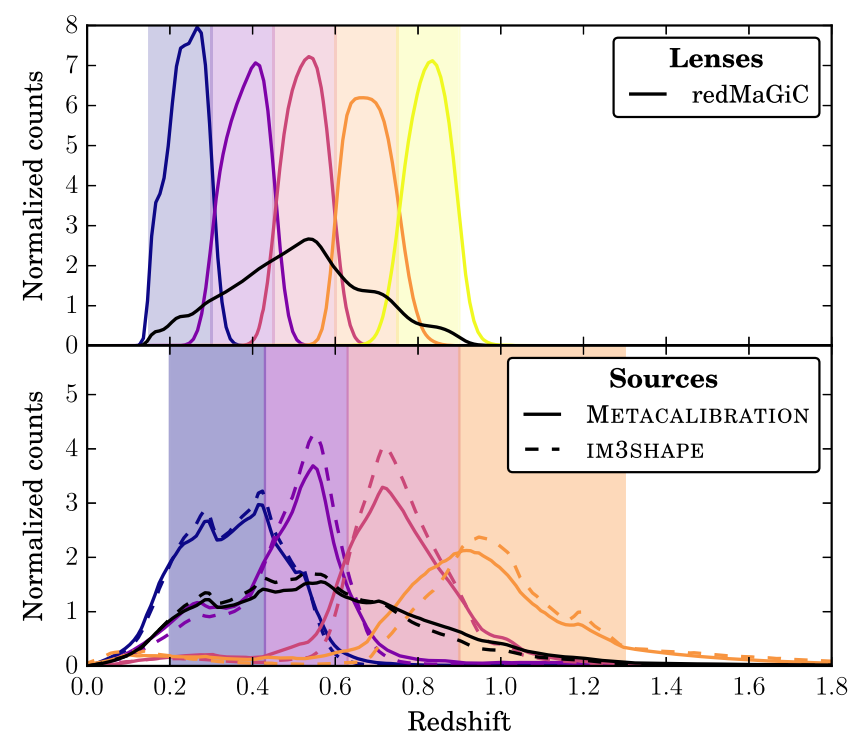

FIG. 1. Estimated redshift distributions of the lens and source galaxies used in the Y1 analysis. The shaded vertical regions define the bins: galaxies are placed in the bin spanning their mean photo- $z$ estimate. We show both the redshift distributions of galaxies in each bin (colored lines) and their overall redshift distributions (black lines). Note that source galaxies were chosen via two different pipelines IM3SHAPE and METACALIBRATION, so their redshift distributions and total numbers differ (solid vs dashed lines).

contaminated by systematic effects. This requires the shallowest or otherwise irregular or patchy regions of the total $1786 \mathrm{deg}^{2} \mathrm{Y} 1$ area to be masked, leaving a contiguous $1321 \mathrm{deg}^{2}$ as the area for the analysis, the region called "SPT" in Ref. [86]. The mask derived for the lens sample is also applied to the source sample.

\section{B. Source galaxies}

\section{Shapes}

Gravitational lensing shear is estimated from the statistical alignment of shapes of source galaxies, which are selected from the Y1 Gold catalog [86]. In DES Y1, we measure galaxy shapes and calibrate those measurements by two independent and different algorithms, METACALIBRATION and IM3SHAPE, as described in Refs. [87].

METACALIBRATION [101,102] measures shapes by simultaneously fitting a two-dimensional (2D) Gaussian model for each galaxy to the pixel data for all available $r-, i-$, and $z$-band exposures, convolving with the point-spread functions (PSFs) appropriate to each exposure. This procedure is repeated on versions of these images that are artificially sheared, i.e., deconvolved, distorted by a shear operator, and reconvolved by a symmetrized version of the PSF. By means of these, the response of the shape measurement to gravitational shear is measured from the images themselves, an approach encoded in METACALIBRATION. 
METACALIBRATION also includes an algorithm for calibration of shear-dependent selection effects of galaxies, which could bias shear statistics at the few percent level otherwise, by measuring on both unsheared and sheared images all those galaxy properties that are used to select, bin and weight galaxies in the catalog. Details of the practical application of these corrections to our lensing estimators are given in Refs. [87,92,93,102].

IM3SHAPE estimates a galaxy shape by determining the maximum likelihood set of parameters from fitting either a bulge or a disk model to each object's $r$-band observations [103]. The maximum likelihood fit, like the Gaussian fit with METACALIBRATION, provides only a biased estimator of shear. For IM3SHAPE, this bias is calibrated using a large suite of image simulations that resemble the DES Y1 data set closely [87,104].

Potential biases in the inferred shears are quantified by multiplicative shear-calibration parameters $m^{i}$ in each source redshift bin $i$, such that the measured shear $\gamma^{\text {meas }}=\left(1+m^{i}\right) \gamma^{\text {true }}$. The $m^{i}$ are free parameters in the cosmological inferences, using prior constraints on each as determined from the extensive systematic-error analyses in Refs. [87]. These shear-calibration priors are listed in Table I. The overall METACALIBRATION calibration is accurate at the level of $1.3 \%$. This uncertainty is dominated by the impact of neighboring galaxies on shape estimates. For tomographic measurements, the widths of the overall $m^{i}$ prior are increased to yield a per-bin uncertainty in $m^{i}$, to account conservatively for possible correlations of $m^{i}$ between bins (see the Appendices of Refs. [87,88]). This yields the $2.3 \%$ prior per redshift bin shown in Table I. The IM3SHAPE prior is determined with $2.5 \%$ uncertainty for the overall sample (increased to a $3.5 \%$ prior per redshift bin), introduced mostly by imperfections in the image simulations.

In both catalogs, we have applied conservative cuts, for instance on signal-to-noise ratio and size, that reduce the number of galaxies with shape estimates relative to the Y1 Gold input catalog significantly. For METACALIBRATION, we obtain 35 million galaxy shape estimates down to an $r$-band magnitude of $\approx 23$. Of these, 26 million are inside the restricted area and redshift bins of this analysis. Since its calibration is more secure, and its number density is higher than that of IM3SHAPE (see Ref. [87] for details on the catalog cuts and methodology details that lead to this difference in number density), we use the METACALIBRATION catalog for our fiducial analysis.

\section{Photometric redshifts}

Redshift probability distributions are also required for source galaxies in cosmological inferences. For each source galaxy, the probability density that it is at redshift $z$, $p_{\mathrm{BPZ}}(z)$, is obtained using a modified version of the Bayesian Photometric Redshifts (BPZ) algorithm [105], as detailed in [88]. Source galaxies are placed in one of four
TABLE I. Parameters and priors ${ }^{\mathrm{a}}$ used to describe the measured two-point functions. "Flat" denotes a flat prior in the range given, while $\operatorname{Gauss}(\mu, \sigma)$ is a Gaussian prior with mean $\mu$ and width $\sigma$. Priors for the tomographic nuisance parameters $m^{i}$ and $\Delta z^{i}$ have been widened to account for the correlation of calibration errors between bins (see Ref. [88] and its Appendix A). The $\Delta z^{i}$ priors listed are for METACALIBRATION galaxies and BPZ photo- $z$ estimates (see Ref. [88] for other combinations). The parameter $w$ is fixed to -1 in the $\Lambda \mathrm{CDM}$ runs.

\begin{tabular}{|c|c|}
\hline Parameter & Prior \\
\hline \multicolumn{2}{|c|}{ Cosmology } \\
\hline$\Omega_{m}$ & Flat $(0.1,0.9)$ \\
\hline$A_{s}$ & Flat $\left(5 \times 10^{-10}, 5 \times 10^{-9}\right)$ \\
\hline$n_{s}$ & Flat $(0.87,1.07)$ \\
\hline$\Omega_{b}$ & Flat $(0.03,0.07)$ \\
\hline$h$ & Flat $(0.55,0.91)$ \\
\hline$\Omega_{\nu} h^{2}$ & Flat $\left(5 \times 10^{-4}, 10^{-2}\right)$ \\
\hline$w$ & Flat $(-2,-0.33)$ \\
\hline \multicolumn{2}{|c|}{ Lens galaxy bias } \\
\hline$b_{i}(i=1,5)$ & Flat $(0.8,3.0)$ \\
\hline \multirow{2}{*}{\multicolumn{2}{|c|}{$\begin{array}{c}\text { Intrinsic alignment } \\
A_{\mathrm{IA}}(z)=A_{\mathrm{IA}}[(1+z) / 1.62]^{\eta_{\mathrm{IA}}}\end{array}$}} \\
\hline & \\
\hline$A_{\mathrm{IA}}$ & flat $(-5,5)$ \\
\hline$\eta_{\mathrm{IA}}$ & flat $(-5,5)$ \\
\hline \multicolumn{2}{|c|}{ Lens photo- $z$ shift (red sequence) } \\
\hline$\Delta z_{1}^{1}$ & Gauss $(0.008,0.007)$ \\
\hline$\Delta z_{1}^{2}$ & Gauss $(-0.005,0.007)$ \\
\hline$\Delta z_{1}^{3}$ & Gauss $(0.006,0.006)$ \\
\hline$\Delta z_{1}^{4}$ & Gauss $(0.000,0.010)$ \\
\hline$\Delta z_{1}^{5}$ & Gauss $(0.000,0.010)$ \\
\hline \multicolumn{2}{|c|}{ Source photo- $z$ shift } \\
\hline$\Delta z_{\mathrm{s}}^{1}$ & Gauss $(-0.001,0.016)$ \\
\hline$\Delta z_{\mathrm{s}}^{2}$ & Gauss $(-0.019,0.013)$ \\
\hline$\Delta z_{\mathrm{s}}^{3}$ & Gauss $(+0.009,0.011)$ \\
\hline$\Delta z_{\mathrm{s}}^{4}$ & Gauss $(-0.018,0.022)$ \\
\hline \multicolumn{2}{|c|}{ Shear calibration } \\
\hline$m_{\text {METACALIBRATION }}^{i}(i=1,4)$ & Gauss $(0.012,0.023)$ \\
\hline$m_{\mathrm{IM} 3 \mathrm{SHAPE}}^{i}(i=1,4)$ & Gauss $(0.0,0.035)$ \\
\hline
\end{tabular}

${ }^{\mathrm{a}}$ The lens photo- $z$ priors changed slightly after unblinding due to changes in the cross-correlation analysis, as described in [90]; we checked that these changes did not impact our results.

redshift bins, $z=[(0.2-0.43),(0.43-0.63),(0.63-0.9)$, $(0.9-1.3)]$, based upon the mean of their $p_{\mathrm{BPZ}}(z)$ distributions. As described in $[88,92,93]$, in the case of METACALIBRATION, these bin assignments are based upon photo- $z$ estimates derived using photometric measurements made by the METACALIBRATION pipeline in order to allow for the correction of selection effects.

We denote by $n_{\mathrm{PZ}}^{i}(z)$ an initial estimate of the redshift distribution of the $N^{i}$ galaxies in bin $i$ produced by randomly drawing a redshift $z$ from the probability distribution $p_{\mathrm{BPZ}}(z)$ of each galaxy assigned to the bin and then bin all these $N^{i}$ redshifts into a histogram. For this step, we use a BPZ estimate based on the optimal flux measurements from the multiepoch multiobject fitting procedure described in Ref. [86]. 
For both the source and the lens galaxies, uncertainties in the redshift distribution are quantified by assuming that the true redshift distribution $n^{i}(z)$ in bin $i$ is a shifted version of the photometrically derived distribution,

$$
n^{i}(z)=n_{\mathrm{PZ}}^{i}\left(z-\Delta z^{i}\right)
$$

with the $\Delta z^{i}$ being free parameters in the cosmological analyses. Prior constraints on these shift parameters are derived in two ways.

First, we constrain $\Delta z^{i}$ from a matched sample of galaxies in the COSMOS field, as detailed in [88]. Reliable redshift estimates for nearly all DES-selectable galaxies in the COSMOS field are available from 30-band imaging [106]. We select and weight a sample of COSMOS galaxies representative of the DES sample with successful shape measurements based on their color, magnitude, and preseeing size. The mean redshift of this COSMOS sample is our estimate of the true mean redshift of the DES source sample, with statistical and systematic uncertainties detailed in [88]. The sample variance in the best-fit $\Delta z^{i}$ from the small COSMOS field is reduced, but not eliminated, by reweighting the COSMOS galaxies to match the multiband flux distribution of the DES source sample.

Second, the $\Delta z^{i}$ of both lens and source samples are further constrained by the angular cross-correlation of each with a distinct sample of galaxies with well-determined redshifts. The $\Delta z_{1}^{i}$ for the three lowest-redshift lens galaxy samples are constrained by cross-correlation of redMaGiC with spectroscopic redshifts [90] obtained in the overlap of DES Y1 with Stripe 82 of the Sloan Digital Sky Survey. The $\Delta z_{\mathrm{s}}^{i}$ for the three lowest-redshift source galaxy bins are constrained by cross-correlating the sources with the REDMAGIC sample, since the REDMAGIC photometric redshifts are much more accurate and precise than those of the sources [89,91]. The $z<0.85$ limit of the REDMAGIC sample precludes the use of cross-correlation to constrain $\Delta z_{\mathrm{s}}^{4}$, so its prior is determined solely by the reweighted COSMOS galaxies.

For the first three source bins, both methods yield an estimate of $\Delta z_{\mathrm{s}}^{i}$, and the two estimates are compatible, so we combine them to obtain a joint constraint. The priors derived for both lens and source redshifts are listed in Table I. The resulting estimated redshift distributions are shown in Fig. 1.

Reference [88] and Fig. 20 in Appendix B demonstrate that, at the accuracy attainable in DES Y1, the precise shapes of the $n^{i}(z)$ functions have a negligible impact on the inferred cosmology as long as the mean redshifts of every bin, parametrized by the $\Delta z^{i}$, are allowed to vary. As a consequence, the cosmological inferences are insensitive to the choice of photometric-redshift algorithm used to establish the initial $n_{\mathrm{PZ}}^{i}(z)$ of the bins.

\section{TWO-POINT MEASUREMENTS}

We measure three sets of two-point statistics: the autocorrelation of the positions of the REDMAGIC lens galaxies, the cross-correlation of the lens positions with the shear of the source galaxies, and the two-point correlation of the source galaxy-shear field. Each of the three classes of statistics is measured using TREECORR [107] in all pairs of redshift bins of the galaxy samples and in $20 \log$-spaced bins of angular separation $2.5^{\prime}<\theta<250^{\prime}$, although we exclude some of the scales and cross-correlations from our fiducial data vector (see Sec. IV). Figures 2 and 3 show these measurements and our best-fit $\Lambda$ CDM model.

\section{A. Galaxy clustering: $w(\theta)$}

The inhomogeneous distribution of matter in the Universe is traced by galaxies. The overabundance of pairs at angular separation $\theta$ above that expected in a random distribution, $w(\theta)$, is one of the simplest measurements of galaxy clustering. It quantifies the strength and scale dependence of the clustering of galaxies, which in turn reflects the clustering of matter.

The upper panel of Fig. 2 shows the angular correlation function of the REDMAGIC galaxies in the five lens redshift bins described above. As described in Ref. [94], these correlation functions were computed after quantifying and correcting for spurious clustering induced by each of multiple observational variables. Figure 2 shows the data with the error bars set equal to the square root of the diagonal elements of the covariance matrix, but we note that data points in nearby angular bins are highly correlated. Indeed, as can be seen in Fig. 5 of Ref. [84], in the lowest redshift bins, the correlation coefficient between almost all angular bins is close to unity; at higher redshift, the measurements are highly correlated only over the adjacent few angular bins. The solid curve in Fig. 2 shows the bestfit prediction from $\Lambda \mathrm{CDM}$ after fitting to all three two-point functions. In principle, we could also use the angular crosscorrelations between galaxies in different redshift bins in the analysis, but the amount of information in these crossbin two-point functions is quite small and would require substantially enlarging the covariance matrix, so we use only the autocorrelations.

\section{B. Galaxy-galaxy lensing: $\gamma_{t}(\boldsymbol{\theta})$}

The shapes of background source galaxies are distorted by the mass associated with foreground lenses. The characteristic distortion is a tangential shear, with the source galaxy ellipticities oriented perpendicular to the line connecting the foreground and background galaxies. This shear, $\gamma_{t}(\theta)$, is sensitive to the mass associated with the foreground galaxies. On scales much larger than the sizes of parent halos of the galaxies, it is proportional to the lens galaxy bias parameters $b^{i}$ in each lens bin which quantifies the relative clumping of matter and galaxies. The lower 

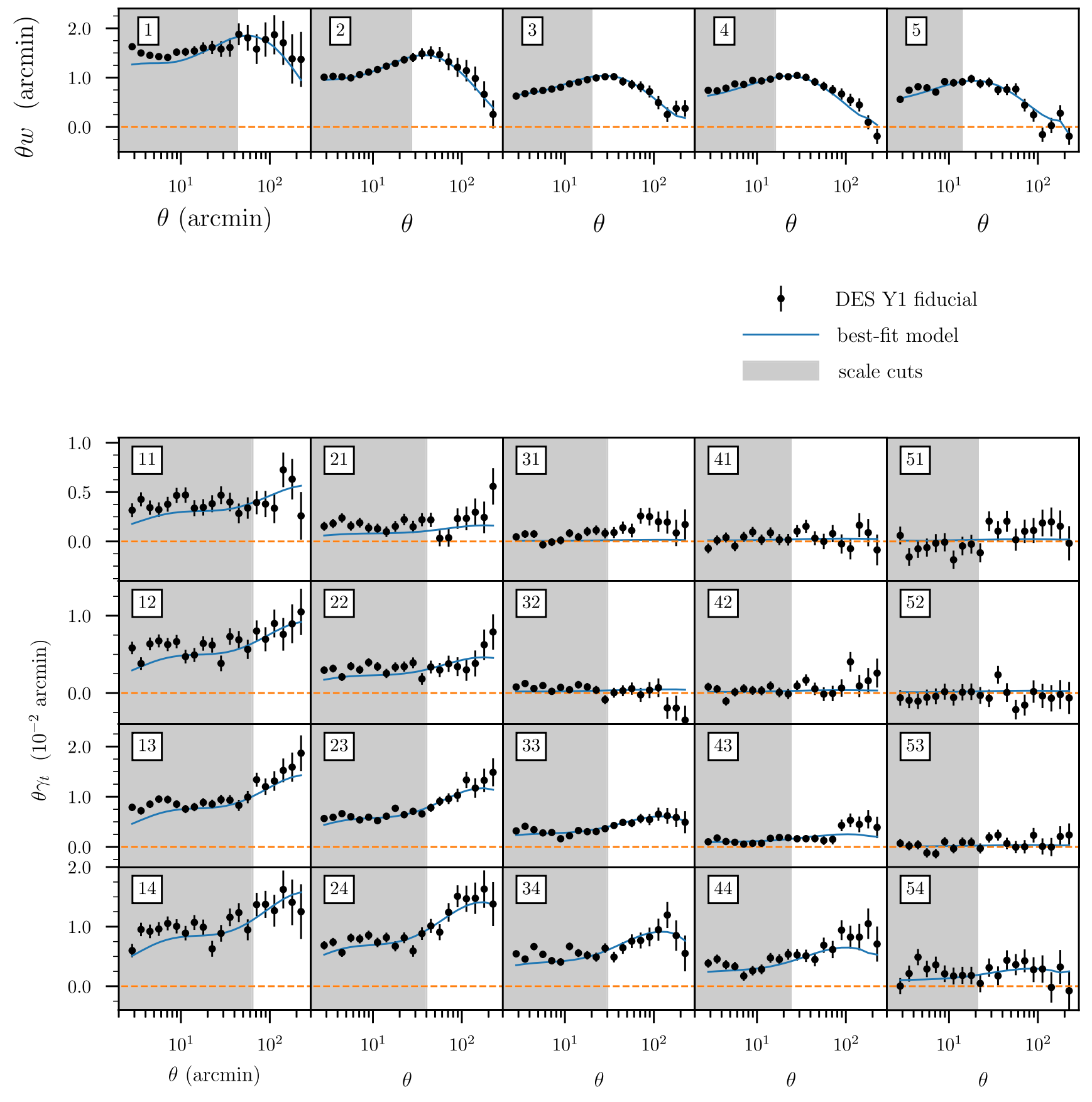

FIG. 2. Top panels: scaled angular correlation function, $\theta w(\theta)$, of REDMAGIC galaxies in the five redshift bins in the top panel of Fig. 1, from lowest (left) to highest redshift (right) [94]. The solid lines are predictions from the $\Lambda$ CDM model that provides the best fit to the combined three two-point functions presented in this paper. Bottom panels: scaled galaxy-galaxy lensing signal, $\theta \gamma_{t}$ (galaxy-shear correlation), measured in DES Y1 in four source redshift bins induced by lens galaxies in five REDMAGIC bins [93]. Columns represent different lens redshift bins, while rows represent different source redshift bins, so, e.g., the bin labeled 12 is the signal from the galaxies in the second source bin lensed by those in the first lens bin. The solid curves are again our best-fit $\Lambda$ CDM prediction. In all panels, shaded areas display the angular scales that have been excluded from our cosmological analysis (see Sec. IV).

panels of Fig. 2 show the measurements of galaxy-galaxy lensing in all pairs of lens-source tomographic bins, including the model prediction for our best-fit parameters. The plots include bin pairs for which the lenses are nominally behind the sources (those toward the upper right) and so might be expected to have zero signal. Although the signals for these bins are expected to be small, they can still be useful in constraining the intrinsic-alignment parameters in our model (see, e.g., Ref. [108]). 

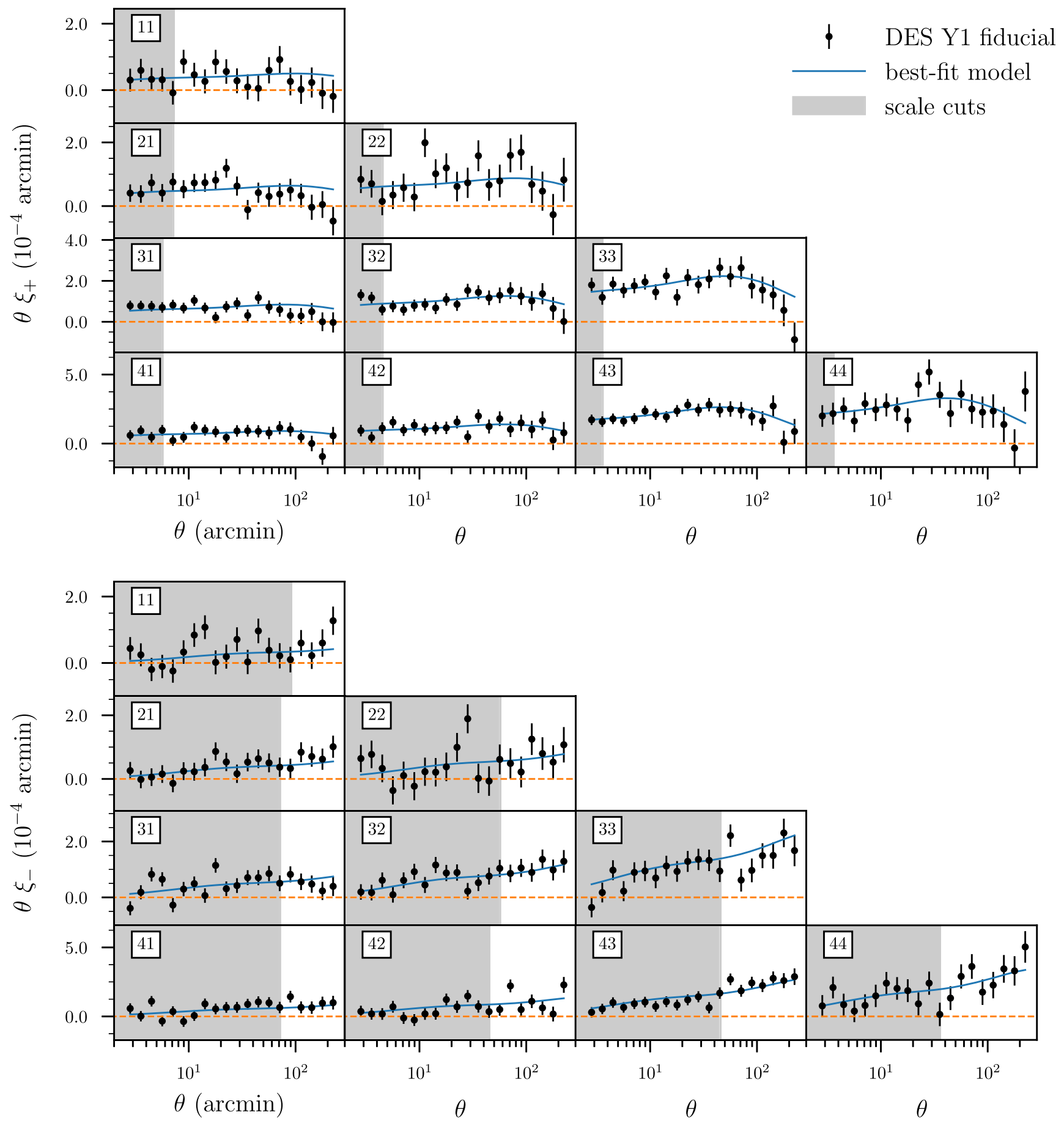

FIG. 3. The cosmic shear correlation functions $\xi_{+}$(top panel) and $\xi_{-}$(bottom panel) in DES Y1 in four source redshift bins, including cross-correlations, measured from the METACALIBRATION shear pipeline (see Ref. [92] for the corresponding plot with IM3SHAPE); pairs of numbers in the upper left of each panel indicate the redshift bins. The solid lines show predictions from our best-fit $\Lambda$ CDM model from the analysis of all three two-point functions, and the shaded areas display the angular scales that are not used in our cosmological analysis (see Sec. IV).

In Ref. [93], we carried out a number of null tests to ensure the robustness of these measurements, none of which showed evidence for significant systematic uncertainties besides the ones characterized by the nuisance parameters in this analysis. The model fits the data well.
Even the fits that appear quite bad are misleading because of the highly off-diagonal covariance matrix. For the nine data points in the 3-1 bin, for example, $\chi^{2}=14$, while $\chi^{2}$ would be 30 if the off-diagonal elements were ignored. 


\section{Cosmic shear: $\boldsymbol{\xi}_{ \pm}(\boldsymbol{\theta})$}

The two-point statistics that quantify correlations between the shapes of galaxies are more complex, because they are the products of the components of a spin-2 tensor. Therefore, a pair of two-point functions is used to capture the relevant information: $\xi_{+}(\theta)$ and $\xi_{-}(\theta)$ are the sum and difference of the products of the tangential and crosscomponents of the shear, measured with respect to the line connecting each galaxy pair. For more details, see Ref. [92] or earlier work in Refs. [109-116]. Figure 3 shows these functions for different pairs of tomographic bins.

As in Fig. 2, the best-fit model prediction here includes the impact of intrinsic alignment, the best-fit shifts in the photometric-redshift distributions, and the best-fit values of shear calibration. The one-dimensional posteriors on all of these parameters are shown in Fig. 19 in Appendix A.

\section{ANALYSIS}

\section{A. Model}

To extract cosmological information from these twopoint functions, we construct a model that depends upon both cosmological parameters and astrophysical and observational nuisance parameters. The cosmological parameters govern the expansion history as well as the evolution and scale dependence of the matter clustering amplitude (as quantified, e.g., by the power spectrum). The nuisance parameters account for uncertainties in photometric redshifts, shear calibration, the bias between galaxies and mass, and the contribution of intrinsic alignment (IA) to the shear spectra. Section IV B will enumerate these parameters, and our priors on them are listed in Table I. Here, we describe how the two-point functions presented in Sec. III are computed in the model.

\section{Galaxy clustering: $w(\theta)$}

Following Ref. [84], we express the projected (angular) density contrast of REDMAGIC galaxies in redshift bin $i$ by $\delta_{\mathrm{g}}^{i}$, the convergence field of source tomography bin $j$ as $\kappa^{j}$, the redshift distribution of the REDMAGIC/source galaxy sample in tomography bin $i$ as $n_{\mathrm{g} / \kappa}^{i}(z)$, and the angular number densities of galaxies in this redshift bin as

$$
\bar{n}_{\mathrm{g} / \kappa}^{i}=\int d z n_{\mathrm{g} / \kappa}^{i}(z) .
$$

The radial weight function for clustering in terms of the comoving radial distance $\chi$ is

$$
q_{\delta_{\mathrm{g}}}^{i}(k, \chi)=b^{i}(k, z(\chi)) \frac{n_{\mathrm{g}}^{i}(z(\chi))}{\bar{n}_{\mathrm{g}}^{i}} \frac{d z}{d \chi},
$$

with $b^{i}(k, z(\chi))$ the galaxy bias of the REDMAGIC galaxies in tomographic bin $i$, and the lensing efficiency

$$
q_{\kappa}^{i}(\chi)=\frac{3 H_{0}^{2} \Omega_{m}}{2 \mathrm{c}^{2}} \frac{\chi}{a(\chi)} \int_{\chi}^{\chi_{\mathrm{h}}} \mathrm{d} \chi^{\prime} \frac{n_{\kappa}^{i}\left(z\left(\chi^{\prime}\right)\right) d z / d \chi^{\prime}}{\bar{n}_{\kappa}^{i}} \frac{\chi^{\prime}-\chi}{\chi^{\prime}},
$$

with $H_{0}$ the Hubble constant, $c$ the speed of light, and $a$ the scale factor. Under the Limber approximation [117-120], the angular correlation function for galaxy clustering can be written as

$$
\begin{aligned}
w^{i}(\theta)= & \int \frac{d l l}{2 \pi} J_{0}(l \theta) \int d \chi \frac{q_{\delta_{\mathrm{g}}}^{i}\left(\frac{l+1 / 2}{\chi}, \chi\right) q_{\delta_{\mathrm{g}}}^{j}\left(\frac{l+1 / 2}{\chi}, \chi\right)}{\chi^{2}} \\
& \times P_{\mathrm{NL}}\left(\frac{l+1 / 2}{\chi}, z(\chi)\right)
\end{aligned}
$$

with $P_{\mathrm{NL}}(k, z)$ the nonlinear matter power spectrum at wave vector $k$ and redshift $z$.

The expression in Eq. (4.4) and the ones in Eqs. (4.5) and (4.6) use the "flat-sky" approximation, which was tested against a curved-sky implementation in Ref. [84] for the case of galaxy clustering. Reference [84] uses the more accurate expression that sums over Legendre polynomials, and we find that these two expressions show negligible differences over the scales of interest.

The model power spectrum here is the fully nonlinear power spectrum in $\Lambda \mathrm{CDM}$ or $w \mathrm{CDM}$, which we estimate on a grid of $(k, z)$ by first running CAMB [121] or CLASS [122] to obtain the linear spectrum and then HALOFIT [123-125] for the nonlinear spectrum. The smallest angular separations for which the galaxy two-point function measurements are used in the cosmological inference, indicated by the boundaries of the shaded regions in the upper panels of Fig. 2, correspond to a comoving scale of $8 h^{-1} \mathrm{Mpc}$; this scale is chosen such that modeling uncertainties in the nonlinear regime cause a negligible impact on the cosmological parameters relative to their statistical errors, as shown in Refs. [84,92].

As described in Sec. VI of Ref. [84], we include the impact of neutrino bias [126-128] when computing the angular correlation function of galaxies. For Y1 data, this effect is below statistical uncertainties, but it is computationally simple to implement and will be relevant for upcoming analyses.

\section{Galaxy-galaxy lensing: $\gamma_{t}(\theta)$}

We model the tangential shear similarly to how we modeled the angular correlation function. Consider the correlation of lens galaxy positions in bin $i$ with source galaxy shear in bin $j$; on large scales, it can be expressed as an integral over the power spectrum,

$$
\begin{aligned}
\gamma_{\mathrm{t}}^{i j}(\theta)= & \left(1+m^{j}\right) \int \frac{d l l}{2 \pi} J_{2}(l \theta) \int d \chi \frac{q_{\delta_{\mathrm{g}}}^{i}\left(\frac{l+1 / 2}{\chi}, \chi\right) q_{\kappa}^{j}(\chi)}{\chi^{2}} \\
& \times P_{\mathrm{NL}}\left(\frac{l+1 / 2}{\chi}, z(\chi)\right),
\end{aligned}
$$


where $m^{j}$ is the multiplicative shear bias and $J_{2}$ is the second-order Bessel function. The shift parameters characterizing the photo- $z$ uncertainties $\Delta z_{\mathrm{s}}^{j}$ and $\Delta z_{1}^{i}$ enter the radial weight functions in Eqs. (4.2) and (4.3) via Eqs. (4.1) and (2.1). The shear signal also depends upon intrinsic alignments of the source shapes with the tidal fields surrounding the lens galaxies; details of our model for this effect (along with an examination of more complex models) are given in Refs. [84,92]. The smallest angular separations for which the galaxy-galaxy lensing measurements are used in the cosmological inference, indicated by the boundaries of the shaded regions in the lower panels of Fig. 2, correspond to a comoving scale of $12 h^{-1} \mathrm{Mpc}$; as above, this scale is chosen such that the model uncertainties in the nonlinear regime cause insignificant changes to the cosmological parameters relative to the statistical uncertainties, as derived in Ref. [84] and verified in Ref. [85].

\section{Cosmic shear $\xi_{ \pm}(\theta)$}

The cosmic shear signal is independent of galaxy bias but shares the same general form as the other sets of twopoint functions. The theoretical predictions for these shearshear two-point functions are

$$
\begin{aligned}
\xi_{+/-}^{i j}(\theta)= & \left(1+m^{i}\right)\left(1+m^{j}\right) \int \frac{d l l}{2 \pi} J_{0 / 4}(l \theta) \\
& \times \int d \chi \frac{q_{\kappa}^{i}(\chi) q_{\kappa}^{j}(\chi)}{\chi^{2}} P_{\mathrm{NL}}\left(\frac{l+1 / 2}{\chi}, z(\chi)\right),
\end{aligned}
$$

where the efficiency functions are defined above and $J_{0}$ and $J_{4}$ are the Bessel functions for $\xi_{+}$and $\xi_{-}$. Intrinsic alignment affects the cosmic shear signal, especially the low-redshift bins, and is modeled as in Ref. [84]. Baryons affect the matter power spectrum on small scales, and the cosmic shear signal is potentially sensitive to these uncertain baryonic effects; we restrict our analysis to the unshaded, large-scale regions shown in Fig. 3 to reduce uncertainty in these effects below our measurement errors, following the analysis in Ref. [92].

\section{B. Parameterization and priors}

We use these measurements from the DES Y1 data to estimate cosmological parameters in the context of two cosmological models, $\Lambda \mathrm{CDM}$ and $w \mathrm{CDM}$. $\Lambda \mathrm{CDM}$ contains three energy densities in units of the critical density: the matter; baryon; and massive neutrino energy densities, $\Omega_{m}, \Omega_{b}$, and $\Omega_{\nu}$. The energy density in massive neutrinos is a free parameter but is often fixed in cosmological analyses to either zero or to a value corresponding to the minimum allowed neutrino mass of $0.06 \mathrm{eV}$ from oscillation experiments [129]. We think it is more appropriate to vary this unknown parameter, and we do so throughout the paper (except in Sec. VII D, where we show that this does not affect our qualitative conclusions). We split the mass equally among the three eigenstates, hence assuming a degenerate mass hierarchy for the neutrinos. Since most other survey analyses have fixed $\Omega_{\nu}$, our results for the remaining parameters will differ slightly from theirs, even when using their data.

$\Lambda \mathrm{CDM}$ has three additional free parameters: the Hubble parameter, $H_{0}$, and the amplitude and spectral index of the primordial scalar density perturbations, $A_{s}$ and $n_{s}$. This model is based on inflation, which fairly generically predicts a flat universe. Further, when curvature is allowed to vary in $\Lambda \mathrm{CDM}$, it is constrained by a number of experiments to be very close to zero. Therefore, although we plan to study the impact of curvature in future work, in this paper, we assume the Universe is spatially flat, with $\Omega_{\Lambda}=1-\Omega_{m}$. It is common to replace $A_{s}$ with the rms amplitude of mass fluctuations on $8 h^{-1} \mathrm{Mpc}$ scale in linear theory, $\sigma_{8}$, which can be derived from the aforementioned parameters. Instead of $\sigma_{8}$, in this work, we will focus primarily on the related parameter

$$
S_{8} \equiv \sigma_{8}\left(\frac{\Omega_{m}}{0.3}\right)^{0.5}
$$

since $S_{8}$ is better constrained than $\sigma_{8}$ and is largely uncorrelated with $\Omega_{m}$ in the DES parameter posterior.

We also consider the possibility that the dark energy is not a cosmological constant. Within this $w \mathrm{CDM}$ model, the dark energy equation-of-state parameter, $w$ [not to be confused with the angular correlation function $w(\theta)$ ], is taken as an additional free parameter instead of being fixed at $w=-1$ as in $\Lambda$ CDM. $w C D M$ thus contains seven cosmological parameters. In future analyses of larger DES data sets, we anticipate constraining more extended cosmological models, e.g., those in which $w$ is allowed to vary in time.

In addition to the cosmological parameters, our model for the data contains 20 nuisance parameters, as indicated in the lower portions of Table I. These are the nine shift parameters, $\Delta z^{i}$, for the source and lens redshift bins; the five REDMAGIC bias parameters, $b^{i}$; the four multiplicative shear biases, $m^{i}$; and two parameters, $A_{\mathrm{IA}}$ and $\eta_{\mathrm{IA}}$, that parametrize the intrinsic-alignment model.

Table I presents the priors we impose on the cosmological and nuisance parameters in the analysis. For the cosmological parameters, we generally adopt flat priors that span the range of values well beyond the uncertainties reported by recent experiments. As an example, although there are currently potentially conflicting measurements of $h$, we choose the lower end of the prior to be $10 \sigma$ below the lower central value from the Planck cosmic microwave background measurement [53] and the upper end to be $10 \sigma$ above the higher central value from local measurements [130]. In the case of $w \mathrm{CDM}$, we impose a physical upper bound of $w<-0.33$, as that is required to obtain cosmic acceleration. As another example, the lower bound of the 
prior on the massive neutrino density, $\Omega_{\nu} h^{2}$, in Table I corresponds to the experimental lower limit on the sum of neutrino masses from oscillation experiments.

For the astrophysical parameters $b^{i}, A_{\mathrm{IA}}$, and $\eta_{\mathrm{IA}}$ that are not well constrained by other analyses, we also adopt conservatively wide, flat priors. For all of these relatively uninformative priors, the guiding principle is that they should not impact our final results and in particular that the tails of the posterior parameter distributions should not lie close to the edges of the priors. ${ }^{3}$ For the remaining nuisance parameters, $\Delta z^{i}$ and $m^{i}$, we adopt Gaussian priors that result from the comprehensive analyses described in Refs. [87-91]. The prior and posterior distributions of these parameters are plotted in Appendix A in Fig. 19.

In evaluating the likelihood function (Sec. IV C), the parameters with Gaussian priors are allowed to vary over a range roughly five times wider than the prior; for example, the parameter that accounts for a possible shift in the furthest lens redshift bin, $\Delta z_{1}^{5}$, has a $1 \sigma$ uncertainty of 0.01 , so it is allowed to vary over $\left|\Delta z_{1}^{5}\right|<0.05$. These sampling ranges conservatively cover the parameter values of interest while avoiding computational problems associated with exploring parameter ranges that are overly broad. Furthermore, overly broad parameter ranges would distort the computation of the Bayesian evidence, which would be problematic as we will use Bayes factors to assess the consistency of the different two-point function measurements, consistency with external data sets, and the need to introduce additional parameters (such as $w$ ) into the analysis. We have verified that our results below are insensitive to the prior ranges chosen.

\section{Likelihood analysis}

For each data set, we sample the likelihood, assumed to be Gaussian, in the many-dimensional parameter space,

$$
\ln \mathcal{L}(\vec{p})=-\frac{1}{2} \sum_{i j}\left[D_{i}-T_{i}(\vec{p})\right] C^{-1}{ }_{i j}\left[D_{j}-T_{j}(\vec{p})\right],
$$

where $\vec{p}$ is the full set of parameters, $D_{i}$ are the measured two-point function data presented in Figs. 2 and 3, and $T_{i}(\vec{p})$ are the theoretical predictions as given in Eqs. (4.4), (4.5), (4.6). The likelihood depends upon the covariance matrix $C$ that describes how the measurement in each angular and redshift bin is correlated with every other measurement. Since the DES data vector contains 457 elements, the covariance is a symmetric $457 \times 457$ matrix. We generate the covariance matrices using CosmoLike [131], which computes the relevant four-point functions in

\footnotetext{
${ }^{3}$ The sole exception is the intrinsic-alignment parameter $\eta_{\mathrm{IA}}$ for which the posterior does hit the edge of the (conservatively selected, given feasible IA evolution) prior; see Fig. 19 in Appendix A.
}

the halo model, as described in Ref. [84]. We also describe there how the CosmoLiKe-generated covariance matrix is tested with simulations.

Equation (4.8) leaves out the $\ln (\operatorname{det}(C))$ in the prefactor ${ }^{4}$ and more generally neglects the cosmological dependence of the covariance matrix. Previous work [132] has shown that this dependence is likely to have a small impact on the central value; our rough estimates of the impact of neglecting the determinant confirm this, and-as we will show belowour results did not change when we replaced the covariance matrix with an updated version based on the best-fit parameters. However, as we will see, the uncertainty in the covariance matrix leads to some lingering uncertainty in the error bars. To form the posterior, we multiply the likelihood by the priors, $\mathcal{P}(\vec{p})$, as given in Table I.

Parallel pipelines, CosmoSIS ${ }^{5}$ [133] and CosmoLike, are used to compute the theoretical predictions and to generate the Monte Carlo Markov chain (MCMC) samples that map out the posterior space leading to parameter constraints. The two sets of software use the publicly available samplers MultiNest [134] and EMCEE [135]. The former provides a powerful way to compute the Bayesian evidence described below, so most of the results shown here use CosmoSIS running MultiNeST.

\section{Tests on simulations}

The collaboration has produced a number of realistic mock catalogs for the DES Y1 data set, based upon two different cosmological $N$-body simulations (Buzzard [136] and MICE [137]), which were analyzed as described in Ref. [85]. We applied all the steps of the analysis on the simulations, from measuring the relevant two-point functions to extracting cosmological parameters. In the case of simulations, the true cosmology is known, and Ref. [85] demonstrates that the analysis pipelines we use here do indeed recover the correct cosmological parameters.

\section{BLINDING AND VALIDATION}

The small statistical uncertainties afforded by the Y1 data set present an opportunity to obtain improved precision on cosmological parameters but also a challenge to avoid confirmation biases. To preclude such biases, we followed the guiding principle that decisions on whether the data analysis has been successful should not be based upon whether the inferred cosmological parameters agreed with our previous expectations. We remained blind to the cosmological parameters implied by the data until after the analysis procedure and estimates of uncertainties on various

\footnotetext{
${ }^{4}$ However, this factor is important for the Bayesian evidence calculations discussed below and so is included in those calculations.

${ }^{5}$ https://bitbucket.org/joezuntz/cosmosis/.
} 
measurement and astrophysical nuisance parameters were frozen.

To implement this principle, we first transformed the ellipticities $e$ in the shear catalogs according to $\operatorname{arctanh}|e| \rightarrow \lambda \operatorname{arctanh}|e|$, where $\lambda$ is a fixed blind random number between 0.9 and 1.1. Second, we avoided plotting the measured values and theoretical predictions in the same figure (including simulation outputs as "theory"). Third, when running codes that derived cosmological parameter constraints from observed statistics, we shifted the resulting parameter values to obscure the best-fit values and/or omitted axis labels on any plots.

These measures were all kept in place until the following criteria were satisfied:

(1) All noncosmological systematics tests of the shear measurements were passed, as described in Refs. [87], and the priors on the multiplicative biases were finalized.

(2) Photo- $z$ catalogs were finalized and passed internal tests, as described in Refs. [88-91].

(3) Our analysis pipelines and covariance matrices, as described in Refs. [84,85], passed all tests, including robustness to intrinsic alignment and bias model assumptions.

(4) We checked that the $\Lambda \mathrm{CDM}$ constraints (on, e.g., $\Omega_{m}, \sigma_{8}$ ) from the two different cosmic shear pipelines IM3SHAPE and METACALIBRATION agreed. The pipelines were not tuned in any way to force agreement.

(5) $\Lambda \mathrm{CDM}$ constraints were stable when dropping the smallest angular bins for METACALIBRATION cosmic shear data.

(6) Small-scale METACALIBRATION galaxy-galaxy lensing data were consistent between source bins (shearratio test, as described in Sec. 6 of Ref. [93]). We note that, while this test is performed in the nominal $\Lambda \mathrm{CDM}$ model, it is close to insensitive to cosmological parameters and therefore does not introduce confirmation bias.

Once the above tests were satisfied, we unblinded the shear catalogs but kept cosmological parameter values blinded while carrying out the following checks, the details of which can be found in Appendix A:

(7) Consistent results were obtained from the two theory/inference pipelines, CosmoSIS and CosMOLIKE.

(8) Consistent results on all cosmological parameters were obtained with the two shear measurement pipelines, METACALIBRATION and IM3SHAPE.

(9) Consistent results on the cosmological parameters were obtained when we dropped the smallest-angular-scale components of the data vector, reducing our susceptibility to baryonic effects and departures from linear galaxy biasing. This test uses the combination of the three two-point functions (as opposed to from shear only as in test 5).
(10) An acceptable goodness-of-fit value $\left(\chi^{2}\right)$ was found between the data and the model produced by the best-fitting parameters. This assured us that the data were consistent with some point in the model space that we are constraining, while not yet revealing which part of parameter space that is.

(11) Parameters inferred from cosmic shear $\left(\xi_{ \pm}\right)$were consistent with those inferred from the combination of galaxy-galaxy lensing $\left(\gamma_{t}\right)$ and galaxy clustering $(w(\theta))$.

Once these tests were satisfied, we unblinded the parameter inferences. The following minor changes to the analysis procedures or priors were made after the unblinding: as planned before unblinding, we reran the MCMC chains with a new covariance matrix calculated at the best-fit parameters of the original analysis. This did not noticeably change the constraints (see Fig. 21 in Appendix B), as expected from our earlier tests on simulated data [84]. We also agreed before unblinding that we would implement two changes after unblinding: small changes to the photo- $z$ priors referred to in the footnote to Table I and fixing a bug in IM3SHAPE object blacklisting that affected $\approx 1 \%$ of the footprint.

All of the above tests passed, most with reassuringly unremarkable results; more details are given in Appendix A.

For test 10 , we calculated the $\chi^{2}(=-2 \log L)$ value of the 457 data points used in the analysis using the full covariance matrix. In $\Lambda \mathrm{CDM}$, the model used to fit the data has 26 free parameters, so the number of degrees of freedom is $\nu=431$. The model is calculated at the bestfit parameter values of the posterior distribution (i.e., the point from the posterior sample with lowest $\chi^{2}$ ). Given the uncertainty on the estimates of the covariance matrix, the formal probabilities of a $\chi^{2}$ distribution are not applicable. We agreed to unblind as long as $\chi^{2}$ was less than $605\left(\chi^{2} / \nu<1.4\right)$. The best-fit value $\chi^{2}=497$ passes this test, ${ }^{6}$ with $\chi^{2} / \nu=1.16$. Considering the fact that 13 of the free parameters are nuisance parameters with tight Gaussian priors, we will use $\nu=444$, giving $\chi^{2} / \nu=1.12$.

The best-fit models for the three two-point functions are plotted over the data in Figs. 2 and 3, from which it is apparent that the $\chi^{2}$ is not dominated by conspicuous outliers. Figure 4 offers confirmation of this, in the form of a histogram of the differences between the best-fit theory and the data in units of the standard deviation of individual data points. The three probes show similar values of $\chi^{2} / \nu$ :

\footnotetext{
${ }^{6}$ In our original analysis (submitted to the arXiv in August 2017), we originally found $\chi^{2}=572$, which passed the aforementioned criterion $\left(\chi^{2}<605\right)$ with proceeding in the analysis. We have since identified a couple of missing ingredients in our computation of the covariance matrix, leading to the present, lower, value $\chi^{2}=497$. While the chi squared has significantly decreased, the cosmological constraints are nearly unchanged. Please see Appendix C for more details.
} 


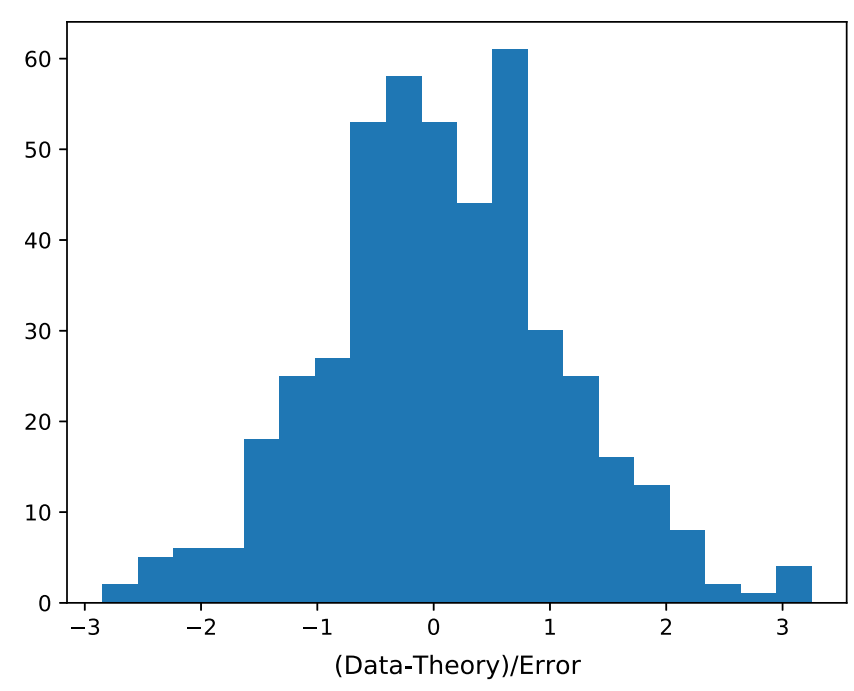

FIG. 4. Histogram of the differences between the best-fit $\Lambda \mathrm{CDM}$ model predictions and the 457 data points shown in Figs. 2 and 3, in units of the standard deviation of the individual data points. Although the covariance matrix is not diagonal, and thus the diagonal error bars do not tell the whole story, it is clear that there are no large outliers that drive the fits.

for $\xi_{ \pm}(\theta), \chi^{2}=230$ for 227 data points; for $\gamma_{t}(\theta), \chi^{2}=185$ for 176 data points; and for $w(\theta), \chi^{2}=68$ for 54 data points. A finer division into each of the 45 individual twopoint functions shows no significant concentration of $\chi^{2}$ in particular bin pairs. We also find that removing all data at scales $\theta>100^{\prime}$ yields $\chi^{2}=278$ for 277 data points $\left(\chi^{2} / \nu=1.05\right)$, not a significant reduction, and also yields no significant shift in best-fit parameters. Thus, we find that no particular piece of our data vector dominates our $\chi^{2}$ result.

Finally, for step number 11 in the test list near the beginning of this section, we examined several measures of consistency between (i) cosmic shear and (ii) $\gamma_{t}(\theta)+w(\theta)$ in $\Lambda \mathrm{CDM}$. As an initial test, we computed the mean of the one-dimensional (1D) posterior distribution of each of the cosmological parameters and measured the shift between $i$ and ii. We then divided this difference by the expected standard deviation of this difference (taking into account the estimated correlation between the $\xi_{ \pm}$and $\gamma_{t}+w$ inferences), $\sigma_{\text {diff }}=\left[\sigma_{\xi_{ \pm}}^{2}+\sigma_{\gamma_{t} w}^{2}-2 \operatorname{Cov}\left(\xi_{ \pm}, \gamma_{t}+w\right)\right]^{1 / 2}$. For all parameters, these differences had absolute value $<0.4$, indicating consistency well within measurement error.

For a second consistency check, we compared the posteriors for the nuisance parameters from cosmic shear to those from clustering plus galaxy-galaxy lensing, and they agreed well. We found no evidence that any of the nuisance parameters push against the edge of its prior or that the nuisance parameters for cosmic shear and $w+\gamma_{t}$ are pushed to significantly different values. The only mild exceptions are modest shifts in the intrinsic alignment parameters, $A_{\mathrm{IA}}$ and $\eta_{\mathrm{IA}}$, as well as in the second source redshift bin, $\Delta z_{s}^{2}$. The full set of posteriors on all 20 nuisance parameters for METACALIBRATION is shown in Fig. 19 in Appendix A.

For a final test of consistency between the two sets of two-point-function measurements, we use the Bayes factor (also called the "evidence ratio"). The Bayes factor is used for discriminating between two hypotheses and is the ratio of the Bayesian evidences, $P(\vec{D} \mid H)$ (the probability of observing data set $\vec{D}$ given hypothesis $H$ ) for each hypothesis. An example of such a hypothesis is that data set $\vec{D}$ can be described by a model $M$, in which case the Bayesian evidence is

$$
P(\vec{D} \mid H)=\int d^{N} \theta P(\vec{D} \mid \vec{\theta}, M) P(\vec{\theta} \mid M),
$$

where $P(\vec{D} \mid \vec{\theta}, M)$ is the likelihood of the data given the model $M$ parametrized by its $N$ parameters $\vec{\theta}$ and $P(\vec{\theta} \mid M)$ is the prior probability distribution of those model parameters.

For two hypotheses $H_{0}$ and $H_{1}$, the Bayes factor is given by

$$
R=\frac{P\left(\vec{D} \mid H_{0}\right)}{P\left(\vec{D} \mid H_{1}\right)}=\frac{P\left(H_{0} \mid \vec{D}\right) P\left(H_{1}\right)}{P\left(H_{1} \mid \vec{D}\right) P\left(H_{0}\right)},
$$

where the second equality follows from Bayes' theorem and clarifies the meaning of the Bayes factor: if we have equal a priori belief in $H_{0}$ and $H_{1}$ [i.e., $P\left(H_{0}\right)=P\left(H_{1}\right)$ ], the Bayes factor is the ratio of the posterior probability of $H_{0}$ to the posterior probability of $H_{1}$. The Bayes factor can be interpreted in terms of odds; i.e., it implies $H_{0}$ is favored over $H_{1}$ with $R: 1$ odds (or disfavored if $R<1$ ). We will adopt the widely used Jeffreys scale [138] for interpreting Bayes factors: $3.2<R<10$ and $R>10$ are respectively considered substantial and strong evidence for $H_{0}$ over $H_{1}$. Conversely, $H_{1}$ is strongly favored over $H_{0}$ if $R<0.1$, and there is substantial evidence for $H_{1}$ if $0.1<R<0.31$.

We follow Ref. [139] by applying this formalism as a test for consistency between cosmological probes. In this case, the null hypothesis, $H_{0}$, is that the two data sets were measured from the same universe and therefore share the same model parameters. Two probes would be judged discrepant if they strongly favor the alternative hypothesis, $H_{1}$, that they are measured from two different universes with different model parameters. So, the appropriate Bayes factor for judging the consistency of two data sets, $D_{1}$ and $D_{2}$, is

$$
R=\frac{P\left(\vec{D}_{1}, \vec{D}_{2} \mid M\right)}{P\left(\vec{D}_{1} \mid M\right) P\left(\vec{D}_{2} \mid M\right)},
$$

where $M$ is the model, e.g., $\Lambda \mathrm{CDM}$ or $w \mathrm{CDM}$. The numerator is the evidence for both data sets when model $M$ is fit to both data sets simultaneously. The denominator is the evidence for both data sets when model $\mathrm{M}$ is fit to both data sets individually, and therefore each data set determines its own parameter posteriors. 
Before the data were unblinded, we decided that we would combine results from these two sets of two-point functions if the Bayes factor defined in Eq. (5.3) did not suggest strong evidence for inconsistency. According to the Jeffreys scale, our condition to combine is therefore that $R>0.1$ (since $R<0.1$ would imply strong evidence for inconsistency). We find a Bayes factor of $R=583$, an indication that DES Y1 cosmic shear and galaxy clustering plus galaxy-galaxy lensing are consistent with one another in the context of $\Lambda \mathrm{CDM}$.

The DES Y1 data were thus validated as internally consistent and robust to our assumptions before we gained any knowledge of the cosmological parameter values that they imply. Any comparisons to external data were, of course, made after the data were unblinded.

\section{DES Y1 RESULTS: PARAMETER CONSTRAINTS}

\section{A. $\Lambda$ CDM}

We first consider the $\Lambda$ CDM model with six cosmological parameters. The DES data are most sensitive to two cosmological parameters, $\Omega_{m}$ and $S_{8}$, as defined in Eq. (4.7), so for the most part we focus on constraints on these parameters.

Given the demonstrated consistency of cosmic shear with clustering plus galaxy-galaxy lensing in the context of $\Lambda \mathrm{CDM}$ as noted above, we proceed to combine the constraints from all three probes. Figure 5 shows the constraints on $\Omega_{m}$ and $\sigma_{8}$ (bottom panel) and on $\Omega_{m}$ and

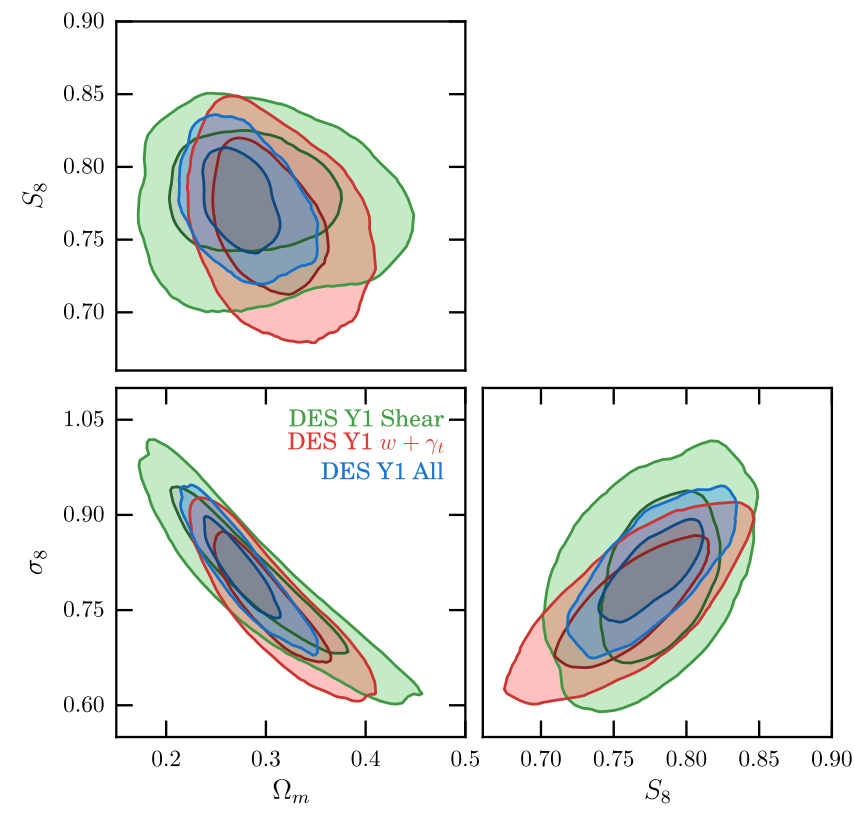

FIG. 5. $\Lambda$ CDM constraints from DES Y1 on $\Omega_{m}, \sigma_{8}$, and $S_{8}$ from cosmic shear (green), REDMAGIC galaxy clustering plus galaxy-galaxy lensing (red), and their combination (blue). Here, and in all such 2D plots below, the two sets of contours depict the $68 \%$ and $95 \%$ confidence levels. the less degenerate parameter $S_{8}$ (top panel). Constraints from cosmic shear, galaxy clustering + galaxy-galaxy lensing, and their combination are shown in these twodimensional subspaces after marginalizing over the 24 other parameters. The combined results lead to constraints

$$
\begin{aligned}
\Omega_{m} & =0.267_{-0.017}^{+0.030} \\
S_{8} & =0.773_{-0.020}^{+0.026} \\
\sigma_{8} & =0.817_{-0.056}^{+0.045} .
\end{aligned}
$$

The value of $\Omega_{m}$ is consistent with the value inferred from either cosmic shear or clustering plus galaxy-galaxy lensing separately. We present the resulting marginalized constraints on the cosmological parameters in the top rows of Table II.

The results shown in Fig. 5, along with previous analyses such as that using KiDS + GAMA data [67], are an important step forward in the capability of combined probes from optical surveys to constrain cosmological parameters. These combined constraints transform what has, for the past decade, been a one-dimensional constraint on $S_{8}$ (which appears banana shaped in the $\Omega_{m}-\sigma_{8}$ plane) into tight constraints on both of these important cosmological parameters. Figure 6 shows the DES Y1 constraints on $S_{8}$ and $\Omega_{m}$ along with some previous results and in combination with external data sets, as will be discussed below. The sizes of these parameter error bars from the combined DES Y1 probes are comparable to those from the CMB obtained by Planck.

In addition to the cosmological parameters, these probes constrain important astrophysical parameters. The intrinsicalignment signal is modeled to scale as $A_{\mathrm{IA}}(1+z)^{\eta_{\mathrm{IA}}}$; while the data do not constrain the power law well $\left(\eta_{\text {IA }}=\right.$ $-0.7 \pm 2.2$ ), they are sensitive to the amplitude of the signal:

$$
A_{\mathrm{IA}}=0.44_{-0.28}^{+0.38} \quad(95 \% \text { C.L. }) .
$$

Further strengthening evidence from the recent combined probes analysis of KiDS $[67,68]$, this result is the strongest evidence to date of IA in a broadly inclusive galaxy sample; previously, significant IA measurements have come from selections of massive elliptical galaxies, usually with spectroscopic redshifts (e.g., Ref. [140]). The ability of DES data to produce such a result without spectroscopic redshifts demonstrates the power of this combined analysis and emphasizes the importance of modeling IA in the pursuit of accurate cosmology from weak lensing. We are able to rule out $A_{\mathrm{IA}}=0$ at $99.76 \%$ C.L. with DES alone and at $99.90 \%$ C.L. with the full combination of DES and external data sets. The mean value of $A_{\mathrm{IA}}$ is nearly the same when combining with external data sets, suggesting that IA self-calibration has been effective. Interestingly, the measured amplitude agrees well with a prediction made by assuming that only red galaxies contribute to the IA signal and then extrapolating the IA amplitude measured from 
TABLE II. $\quad 68 \%$ C.L. marginalized cosmological constraints in $\Lambda$ CDM and $w C D M$ using a variety of data sets. "DES Y1 3x2" refers to results from combining all three two-point functions in DES Y1. Cells with no entries correspond to posteriors not significantly narrower than the prior widths. The only exception is in $w C D M$ for Planck only, where the posteriors on $h$ are shown to indicate the large values inferred in the model without any data to break the $w-h$ degeneracy.

\begin{tabular}{|c|c|c|c|c|c|c|c|c|}
\hline Model & Data sets & $\Omega_{m}$ & $S_{8}$ & $n_{s}$ & $\Omega_{b}$ & $h$ & $\sum_{(95 \% \mathrm{CL})} m_{\nu}(\mathrm{eV})$ & $w$ \\
\hline$\Lambda \mathrm{CDM}$ & DES Y1 $\xi_{ \pm}(\theta)$ & $0.260_{-0.037}^{+0.065}$ & $0.782_{-0.027}^{+0.027}$ & $\cdots$ & $\ldots$ & $\cdots$ & $\cdots$ & $\ldots$ \\
\hline$\Lambda \mathrm{CDM}$ & DES Y1 $w(\theta)+\gamma_{t}$ & $0.288_{-0.026}^{+0.045}$ & $0.760_{-0.030}^{+0.033}$ & $\ldots$ & $\ldots$ & $\cdots$ & $\cdots$ & $\cdots$ \\
\hline$\Lambda \mathrm{CDM}$ & DES Y $13 \times 2$ & $0.267_{-0.017}^{+0.030}$ & $0.773_{-0.020}^{+0.026}$ & $\ldots$ & $\ldots$ & $\ldots$ & $\ldots$ & $\ldots$ \\
\hline$\Lambda \mathrm{CDM}$ & Planck (no lensing) & $0.334_{-0.026}^{+0.037}$ & $0.841_{-0.025}^{+0.027}$ & $0.958_{-0.005}^{+0.008}$ & $0.0503_{-0.0019}^{+0.0046}$ & $0.658_{-0.027}^{+0.019}$ & $\cdots$ & $\cdots$ \\
\hline$\Lambda \mathrm{CDM}$ & DES Y1 + Planck (no lensing) & $0.297_{-0.012}^{+0.016}$ & $0.795_{-0.013}^{+0.020}$ & $0.972_{-0.004}^{+0.006}$ & $0.0477_{-0.0012}^{+0.0016}$ & $0.686_{-0.014}^{+0.009}$ & $<0.47$ & $\ldots$ \\
\hline$\Lambda \mathrm{CDM}$ & $\mathrm{DES} \mathrm{Y} 1+\mathrm{JLA}+\mathrm{BAO}$ & $0.295_{-0.014}^{+0.018}$ & $0.768_{-0.023}^{+0.018}$ & $1.044_{-0.087}^{+0.019}$ & $0.0516_{-0.0080}^{+0.0050}$ & $0.672_{-0.034}^{+0.049}$ & $\ldots$ & $\ldots$ \\
\hline$\Lambda \mathrm{CDM}$ & Planck + JLA + BAO & $0.306_{-0.007}^{+0.007}$ & $0.815_{-0.013}^{+0.015}$ & $0.969_{-0.005}^{+0.004}$ & $0.0483_{-0.0006}^{+0.0008}$ & $0.678_{-0.005}^{+0.007}$ & $<0.22$ & $\ldots$ \\
\hline$\Lambda \mathrm{CDM}$ & DES Y1 + Planck + JLA + BAO & $0.298_{-0.007}^{+0.007}$ & $0.802_{-0.012}^{+0.012}$ & $0.973_{-0.004}^{+0.005}$ & $0.0479_{-0.0008}^{+0.0007}$ & $0.685_{-0.007}^{+0.005}$ & $<0.26$ & $\ldots$ \\
\hline$w \mathrm{CDM}$ & DES Y $1 \xi_{ \pm}(\theta)$ & $0.274_{-0.042}^{+0.073}$ & $0.777_{-0.038}^{+0.036}$ & $\ldots$ & $\ldots$ & $\ldots$ & $\ldots$ & $-0.99_{-0.39}^{+0.33}$ \\
\hline$w \mathrm{CDM}$ & DES Y1 $w(\theta)+\gamma_{t}$ & $0.310_{-0.036}^{+0.049}$ & $0.785_{-0.072}^{+0.040}$ & $\cdots$ & $\ldots$ & $\ldots$ & $\ldots$ & $-0.79_{-0.39}^{+0.22}$ \\
\hline$w \mathrm{CDM}$ & DES Y1 $3 \times 2$ & $0.284_{-0.030}^{+0.033}$ & $0.782_{-0.024}^{+0.036}$ & $\ldots$ & $\ldots$ & $\ldots$ & $\ldots$ & $-0.82_{-0.20}^{+0.21}$ \\
\hline$w \mathrm{CDM}$ & Planck (no lensing) & $0.222_{-0.024}^{+0.069}$ & $0.810_{-0.036}^{+0.029}$ & $0.960_{-0.007}^{+0.005}$ & $0.0334_{-0.0032}^{+0.0099}$ & $0.801_{-0.097}^{+0.045}$ & $\ldots$ & $-1.47_{-0.22}^{+0.31}$ \\
\hline$w \mathrm{CDM}$ & DES Y1 + Planck (no lensing) & $0.233_{-0.033}^{+0.025}$ & $0.775_{-0.021}^{+0.021}$ & $0.971_{-0.006}^{+0.004}$ & $0.0355_{-0.0039}^{+0.0050}$ & $0.775_{-0.040}^{+0.056}$ & $<0.65$ & $-1.35_{-0.17}^{+0.16}$ \\
\hline$w \mathrm{CDM}$ & Planck + JLA + BAO & $0.303_{-0.008}^{+0.010}$ & $0.816_{-0.013}^{+0.014}$ & $0.968_{-0.006}^{+0.004}$ & $0.0479_{-0.0014}^{+0.0016}$ & $0.679_{-0.008}^{+0.013}$ & $<0.27$ & $-1.02_{-0.05}^{+0.05}$ \\
\hline$w \mathrm{CDM}$ & $\mathrm{DES} \mathrm{Y} 1+\mathrm{Planck}+\mathrm{JLA}+\mathrm{BAO}$ & $0.301_{-0.010}^{+0.007}$ & $0.801_{-0.012}^{+0.011}$ & $0.974_{-0.005}^{+0.005}$ & $0.0483_{-0.0016}^{+0.0014}$ & $0.680_{-0.008}^{+0.013}$ & $<0.31$ & $-1.00_{-0.04}^{+0.05}$ \\
\hline
\end{tabular}

spectroscopic samples of luminous galaxies using a realistic luminosity function and red galaxy fraction [84]. Our measurement extends the diversity of galaxies with evidence of IA, allowing more precise predictions for the behavior of the expected IA signal.
The biases of the REDMAGIC galaxy samples in the five lens bins are shown in Fig. 7 along with the results with fixed cosmology obtained in Refs. [93,94]. The biases are measured to be $b_{1}=1.42_{-0.08}^{+0.13}, b_{2}=1.65_{-0.12}^{+0.08}$, $b_{3}=1.60_{-0.08}^{+0.11}, b_{4}=1.92_{-0.10}^{+0.14}$, and $b_{5}=2.00_{-0.14}^{+0.13}$. Even
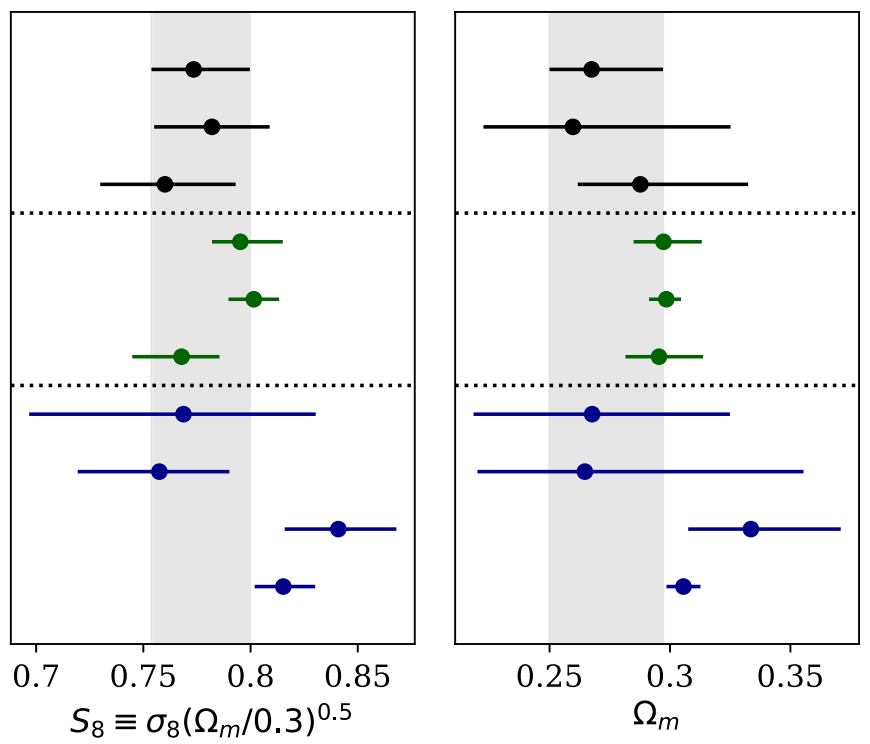

DES Y1 All

DES Y1 Shear

DES Y1 $w+\gamma_{t}$

DES Y1 All + Planck (No Lensing)

DES Y1 All + Planck + BAO + JLA

DES Y1 All + BAO + JLA

DES SV

KiDS-450

Planck (No Lensing)

Planck + BAO + JLA

FIG. 6. $68 \%$ confidence levels for $\Lambda \mathrm{CDM}$ on $S_{8}$ and $\Omega_{m}$ from DES Y1 (different subsets considered in the top group, black), DES Y1 with all three probes combined with other experiments (middle group, green); , and results from previous experiments (bottom group, purple). Note that neutrino mass has been varied, so, e.g., results shown for KiDS-450 were obtained by reanalyzing their data with the neutrino mass left free. The table includes only data sets that are publicly available so that we could reanalyze those using the same assumptions (e.g., free neutrino mass) that are used in our analysis of DES Y1 data. 


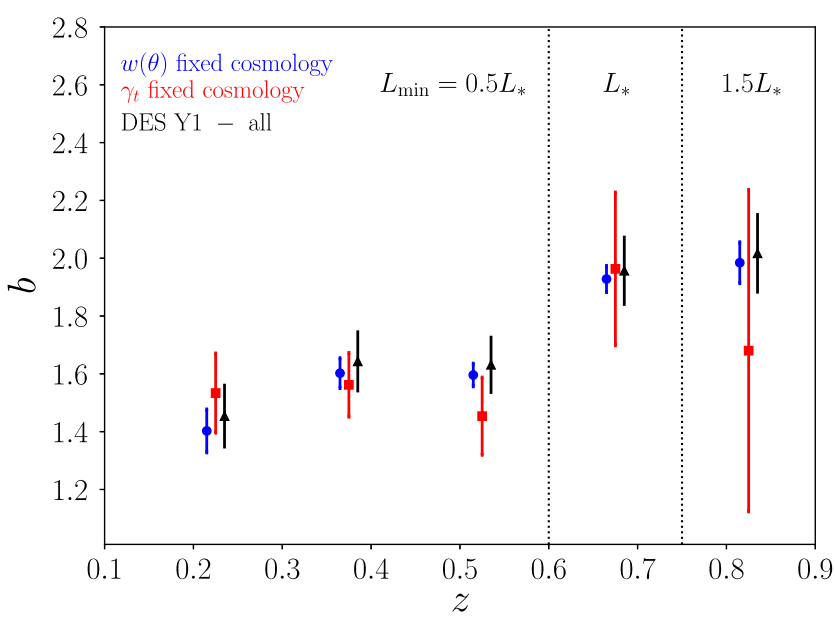

FIG. 7. The bias of the REDMAGIC galaxy samples in the five lens bins from three separate DES Y1 analyses. The two labeled "fixed cosmology" use the galaxy angular correlation function $w(\theta)$ and galaxy-galaxy lensing $\gamma_{t}$, respectively, with cosmological parameters fixed at best-fit values from the $3 \times 2$ analysis, as described in Refs. [93,94]. The results labeled "DES Y1-all" vary all 26 parameters while fitting to all three two-point functions.

when varying a full set of cosmological parameters (including $\sigma_{8}$, which is quite degenerate with bias when using galaxy clustering only) and 15 other nuisance parameters, the combined probes in DES Y1 therefore constrain bias at the $10 \%$ level.

\section{B. $w \mathrm{CDM}$}

A variety of theoretical alternatives to the cosmological constant has been proposed [6]. For example, it could be that the cosmological constant vanishes and that another degree of freedom, e.g., a very light scalar field, is driving the current epoch of accelerated expansion. Here, we restrict our analysis to the simplest class of phenomenological alternatives, models in which the dark energy density is not constant but rather evolves over cosmic history with a constant equation-of-state parameter, $w$. We constrain $w$ by adding it as a seventh cosmological parameter. Here, too, DES obtains interesting constraints on only a subset of the seven cosmological parameters, so we show the constraints on the three-dimensional subspace spanned by $\Omega_{m}, S_{8}$, and $w$. Figure 8 shows the constraints in this three-dimensional space from cosmic shear and from galaxy-galaxy lensing + galaxy clustering. These two sets of probes agree with one another. The consistency in the three-dimensional subspace shown in Fig. 8, along with the tests in the previous subsection, is sufficient to combine the two sets of probes. The Bayes factor in this case is equal to 1878 . The combined constraint from all three two-point functions is also shown in Fig. 8.

The marginalized $68 \%$ C.L. constraints on $w$ and on the other two cosmological parameters tightly constrained by

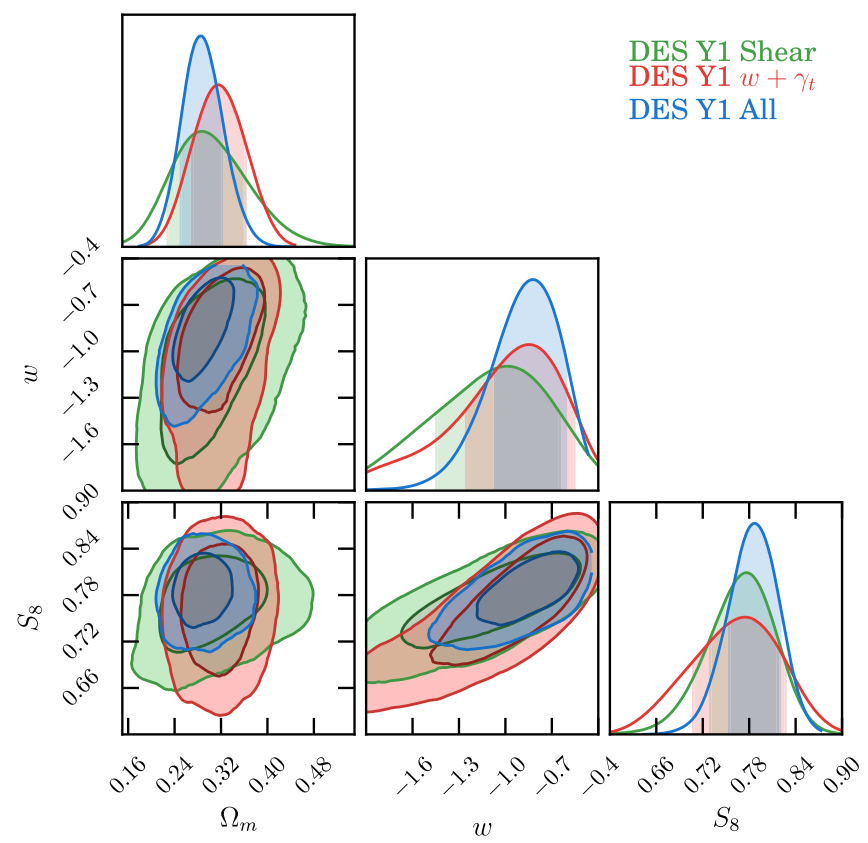

FIG. 8. Constraints on the three cosmological parameters $\sigma_{8}$, $\Omega_{m}$, and $w$ in $w$ CDM from DES Y1 after marginalizing over four other cosmological parameters and 10 (cosmic shear only) or 20 (other sets of probes) nuisance parameters. The constraints from cosmic shear only (green), $w(\theta)+\gamma_{t}(\theta)$ (red), and all three twopoint functions (blue) are shown. Here and below, outlying panels show the marginalized 1D posteriors and the corresponding $68 \%$ confidence regions.

DES, $S_{8}$ and $\Omega_{M}$, are shown in Fig. 9 and given numerically in Table II. In the next section, we revisit the question of how consistent the DES Y1 results are with other experiments. The marginalized constraint on $w$ from all three DES Y1 probes is

$$
w=-0.82_{-0.20}^{+0.21}
$$

Finally, if one ignores any intuition or prejudice about the mechanism driving cosmic acceleration, studying $w \mathrm{CDM}$ translates into adding an additional parameter to describe the data. From a Bayesian point of view, the question of whether $w C D M$ is more likely than $\Lambda \mathrm{CDM}$ can again be addressed by computing the Bayes factor. Here, the two models being compared are simpler: $\Lambda \mathrm{CDM}$ and $w \mathrm{CDM}$. The Bayes factor is

$$
R_{w}=\frac{P(\vec{D} \mid w \mathrm{CDM})}{P(\vec{D} \mid \Lambda \mathrm{CDM})} .
$$

Values of $R_{w}$ less than unity would imply $\Lambda$ CDM is favored, while those greater than 1 argue that the introduction of the additional parameter $w$ is warranted. The Bayes factor is $R_{w}=0.39$ for DES Y 1 , so although $\Lambda \mathrm{CDM}$ 

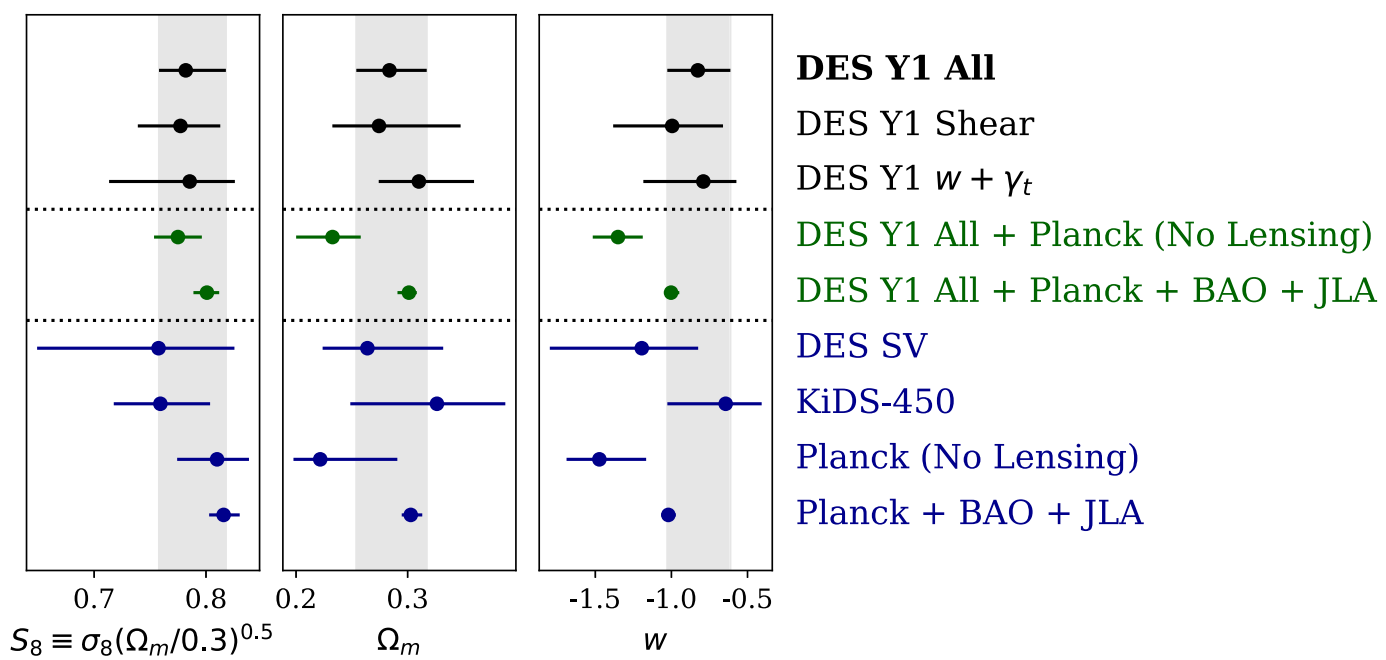

FIG. 9. 68\% confidence levels on three cosmological parameters from the joint DES Y1 probes and other experiments for $w$ CDM.

is slightly favored, there is no compelling evidence to favor or disfavor an additional parameter $w$.

It is important to note that, although our result in Eq. (6.3) is compatible with $\Lambda \mathrm{CDM}$, the most stringent test of the model from DES Y1 is not this parameter but rather the constraints on the parameters in the model shown in Fig. 5 as compared with constraints on those parameters from the CMB measurements of the Universe at high redshift. We turn next to that comparison.

\section{COMPARISON WITH EXTERNAL DATA}

We next explore the cosmological implications of comparison and combination of DES Y1 results with other experiments' constraints. For the CMB, we take constraints from Planck [53]. In the first subsection below, we use only the temperature and polarization auto- and cross-spectra from Planck, omitting the information due to lensing of the CMB that is contained in the four-point function. The latter depends on structure and distances at late times, and we wish in this subsection to segregate late-time information from early-Universe observables. We use the joint TT, EE, BB, and TE likelihood for multipoles $\ell$ between 2 and 29 and the TT likelihood for $\ell$ between 30 and 2508 (commonly referred to as $\mathrm{TT}+$ lowP), provided by Planck. $^{7}$ In all cases that we have checked, the use of WMAP [141] data yields constraints consistent with, but weaker than, those obtained with Planck. Recent results from the South Pole Telescope [142] favor a value of $\sigma_{8}$ that is $2.6 \sigma$ lower than Planck, but we have not yet tried to incorporate these results.

We use measured angular diameter distances from the baryon acoustic oscillation (BAO) feature by the $6 \mathrm{dF}$

\footnotetext{
${ }^{7}$ Late-Universe lensing does smooth the CMB power spectra slightly, so these data sets are not completely independent of low redshift information.
}

Galaxy Survey [143], the SDSS Data Release 7 Main Galaxy Sample [144], and BOSS Data Release 12 [50], in each case extracting only the BAO constraints. These BAO distances are all measured relative to the physical BAO scale corresponding to the sound horizon distance $r_{\mathrm{d}}$; therefore, dependence of $r_{\mathrm{d}}$ on cosmological parameters must be included when determining the likelihood of any cosmological model (see Ref. [50] for details). We also use measures of luminosity distances from observations of distant type Ia supernovae ( $\mathrm{SNe}$ ) via the Joint Lightcurve Analysis (JLA) data from Ref. [145].

This set of $\mathrm{BAO}$ and $\mathrm{SNe}$ experiments has been shown to be consistent with the $\Lambda \mathrm{CDM}$ and $w \mathrm{CDM}$ constraints from the CMB [51,53], so we can therefore sensibly merge this suite of experiments-BAO, $\mathrm{SNe}$, and Planck-with the DES Y1 results to obtain unprecedented precision on the cosmological parameters. We do not include information about direct measurements of the Hubble constant because those are in tension with this bundle of experiments [146].

\section{A. High redshift vs low redshift in $\Lambda \mathrm{CDM}$}

The CMB measures the state of the Universe when it was 380,000 years old, while DES measures the matter distribution in the Universe roughly ten billion years later. Therefore, one obvious question that we can address is as follows: Is the $\Lambda \mathrm{CDM}$ prediction for clustering today, with all cosmological parameters determined by Planck, consistent with what DES observes? This question, which has of course been addressed by previous surveys (e.g., Refs. $[31,35,67,68]$ ), is so compelling because (i) of the vast differences in the epochs and conditions measured; (ii) the predictions for the DES Y1 values of $S_{8}$ and $\Omega_{m}$ have no free parameters in $\Lambda \mathrm{CDM}$ once the recombinationera parameters are fixed; and (iii) those predictions for what DES should observe are very precise, with $S_{8}$ and $\Omega_{m}$ determined by the CMB to within a few percent. We saw 


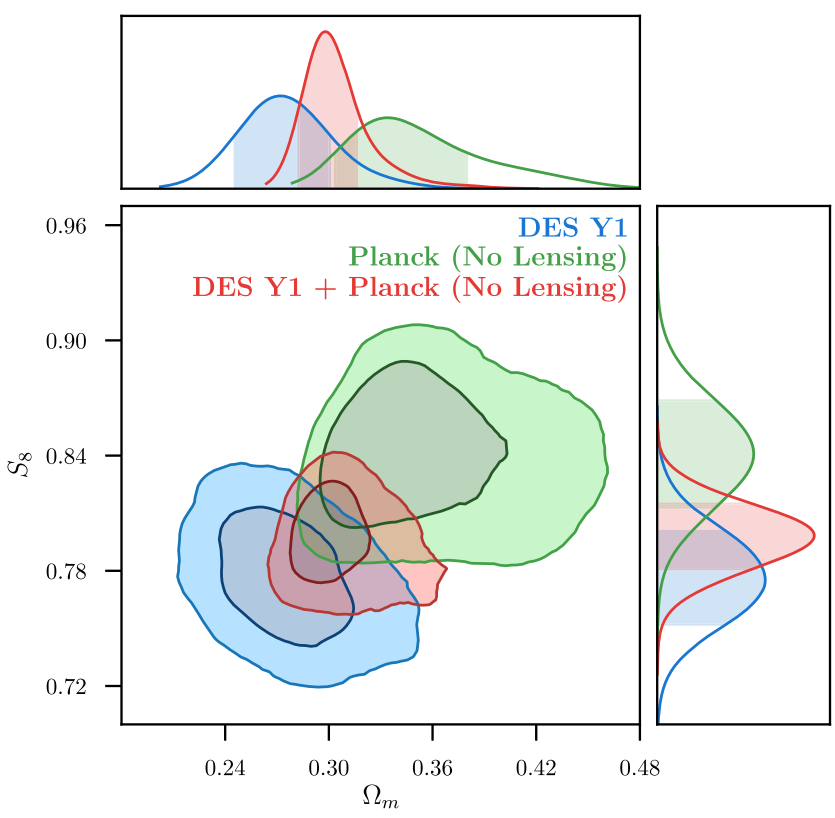

FIG. 10. $\Lambda \mathrm{CDM}$ constraints from the three combined probes in DES Y1 (blue), Planck with no lensing (green), and their combination (red). The agreement between DES and Planck can be quantified via the Bayes factor, which indicates that in the full, multidimensional parameter space the two data sets are consistent (see the text).

above that $S_{8}$ and $\Omega_{m}$ are constrained by DES Y1 at the few-percent level, so the stage is set for the most stringent test yet of $\Lambda \mathrm{CDM}$ growth predictions. Tension between these two sets of constraints might imply the breakdown of $\Lambda \mathrm{CDM}$.

Figure 10 compares the low- $z$ constraints for $\Lambda \mathrm{CDM}$ from all three DES Y1 probes with the $z=1100$ constraints from the Planck anisotropy data. Note that the Planck contours are shifted slightly and widened significantly from those in Fig. 18 of Ref. [53], because we are marginalizing over the unknown sum of the neutrino masses. We have verified that when the sum of the neutrino masses is fixed as Ref. [53] assumed in their fiducial analysis we recover the constraints shown in their Fig. 18.

The two-dimensional constraints shown in Fig. 10 visually hint at tension between the Planck $\Lambda$ CDM prediction for rms mass fluctuations and the matter density of the present-day Universe and the direct determination by DES. The 1D marginal constraints differ by more than $1 \sigma$ in both $S_{8}$ and $\Omega_{m}$, as shown in Fig. 6. The KiDS survey $[35,67,68,147]$ and, earlier, Canada-France Hawaii Telescope Lensing Survey $[31,148]$ also report lower $S_{8}$ than Planck at marginal significance.

However, a more quantitative measure of consistency in the full 26-parameter space is the Bayes factor defined in Eq. (5.3). As mentioned above, a Bayes factor below 0.1 suggests strong inconsistency, and one above 10 suggests strong evidence for consistency. The Bayes factor for combining DES and Planck (no lensing) in the $\Lambda \mathrm{CDM}$ model is $R=6.6$, indicating "substantial" evidence for consistency on the Jeffreys scale, so any inconsistency apparent in Fig. 10 is not statistically significant according to this metric. In order to test the sensitivity of this conclusion to the priors used in our analysis, we halve the width of the prior ranges on all cosmological parameters (the parameters in the first section of Table I). For this case, we find $R=0.75$; despite dropping by nearly a factor of $10, R$ it is still above 0.1 , and therefore we are still passing the consistency test. The Bayes factor in Eq. (5.3) compares the hypothesis that two data sets can be fit by the same set of $N$ model parameters (the null hypothesis) to the hypothesis that they are each allowed an independent set of the $N$ model parameters (the alternative hypothesis). The alternative hypothesis is naturally penalized in the Bayes factor since the model requires an extra $N$ parameters. We also test an alternative hypothesis where only $\Omega_{m}$ and $A_{s}$ are allowed to be constrained independently by the two data sets; in this case, we are introducing only two extra parameters with respect to the null hypothesis. For this case, we find $R=0.47$, which again indicates that there is no evidence for inconsistency between the data sets.

We therefore combine the two data sets, resulting in the red contours in Fig. 10. This quantitative conclusion that the high- and low-redshift data sets are consistent can even be gleaned by viewing Fig. 10 in a slightly different way: if the true parameters lie within the red contours, it is not unlikely for two independent experiments to return the blue and green contour regions.

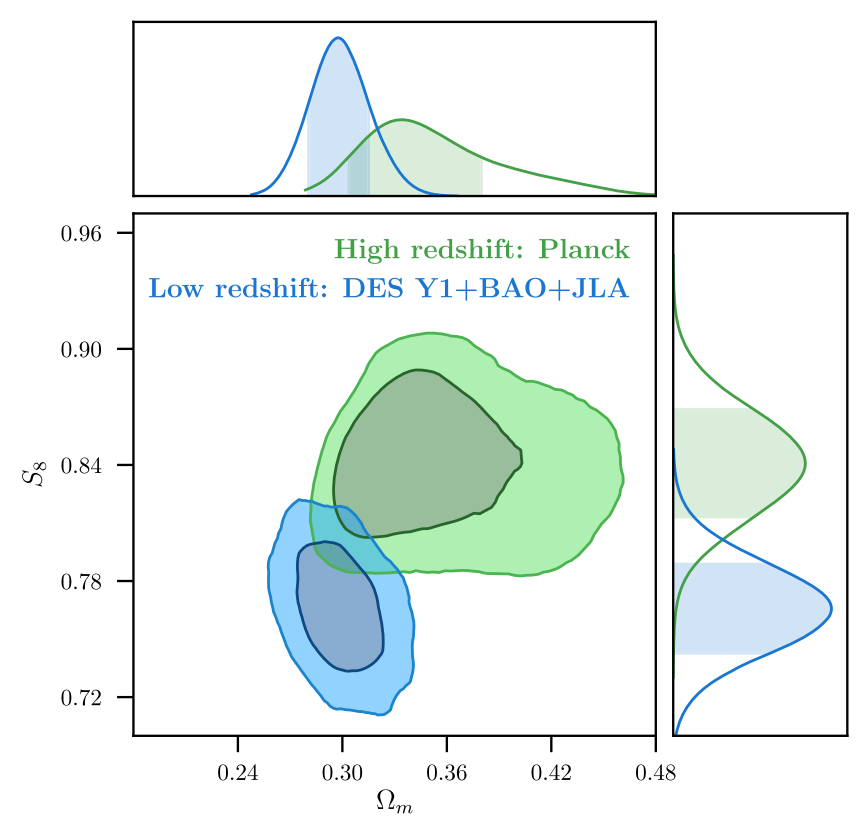

FIG. 11. $\Lambda \mathrm{CDM}$ constraints from high redshift (Planck, without lensing) and multiple low redshift experiments (DES $\mathrm{Y} 1+\mathrm{BAO}+\mathrm{JLA})$; see the text for references. 


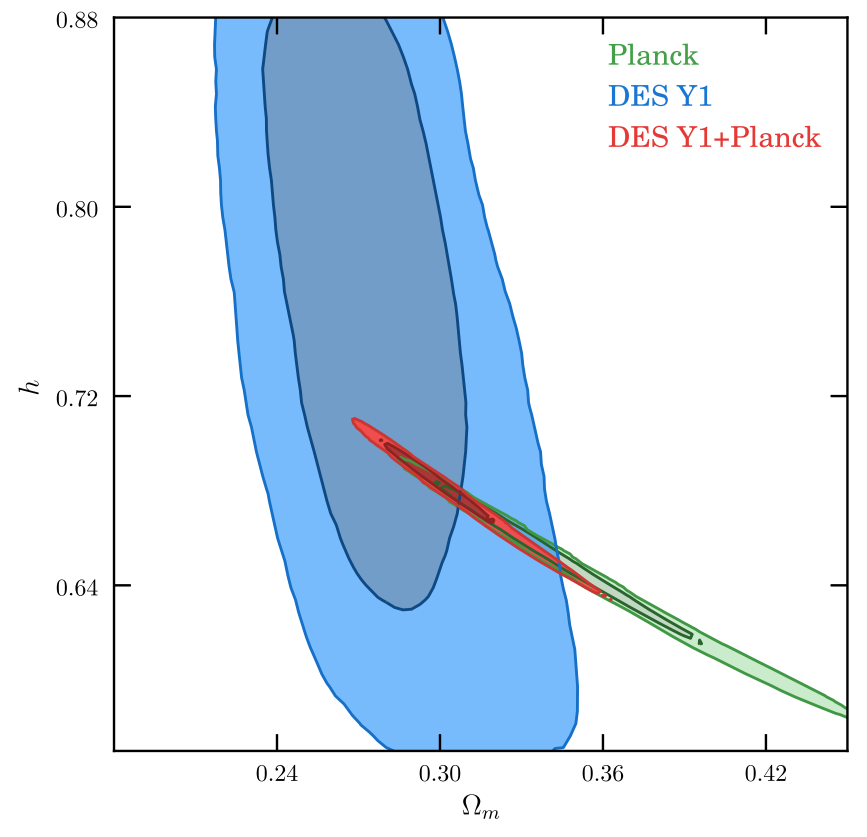

FIG. 12. $\Lambda \mathrm{CDM}$ constraints from Planck with no lensing (green), DES Y1 (blue) and the two combined (red) in the $\Omega_{m}, h$ plane. The positions of the acoustic peaks in the CMB constrain $\Omega_{m} h^{3}$ extremely well, and the DES determination of $\Omega_{m}$ breaks the degeneracy, leading to a larger value of $h$ than inferred from Planck only (see Table II).

Figure 11 takes the high $z$ vs low- $z$ comparison a step further by combining DES Y1 with results from BAO experiments and type Ia supernovae. While these even tighter low-redshift constraints continue to favor slightly lower values of $\Omega_{m}$ and $S_{8}$ than Planck, the Bayes factor is 0.6 , which neither favors nor disfavors the hypothesis that the two sets of data, DES Y1 + BAO + JLA on one hand and Planck on the other, are described by the same set of cosmological parameters.

The goal of this subsection is to test the $\Lambda \mathrm{CDM}$ prediction for clustering in DES, so we defer the issue of parameter determination to the next subsections. However, there is one aspect of the CMB measurements combined with DES that is worth mentioning here. DES data do not constrain the Hubble constant directly. However, as shown in Fig. 12, the DES $\Lambda$ CDM constraint on $\Omega_{m}$ combined with Planck's measurement of $\Omega_{m} h^{3}$ leads to a shift in the inference of the Hubble constant (in the direction of local measurements [130]). Since $\Omega_{m}$ is lower in DES, the inferred value of $h$ moves up. As shown in the figure and quantitatively in Table II, the shift is greater than $1 \sigma$. As shown in Table II, this shift in the value of $h$ persists as more data sets are added in.

\section{B. Cosmological parameters in $\Lambda \mathrm{CDM}$}

To obtain the most stringent cosmological constraints, we now compare DES Y1 with the bundle of BAO, Planck, and JLA that have been shown to be consistent with one

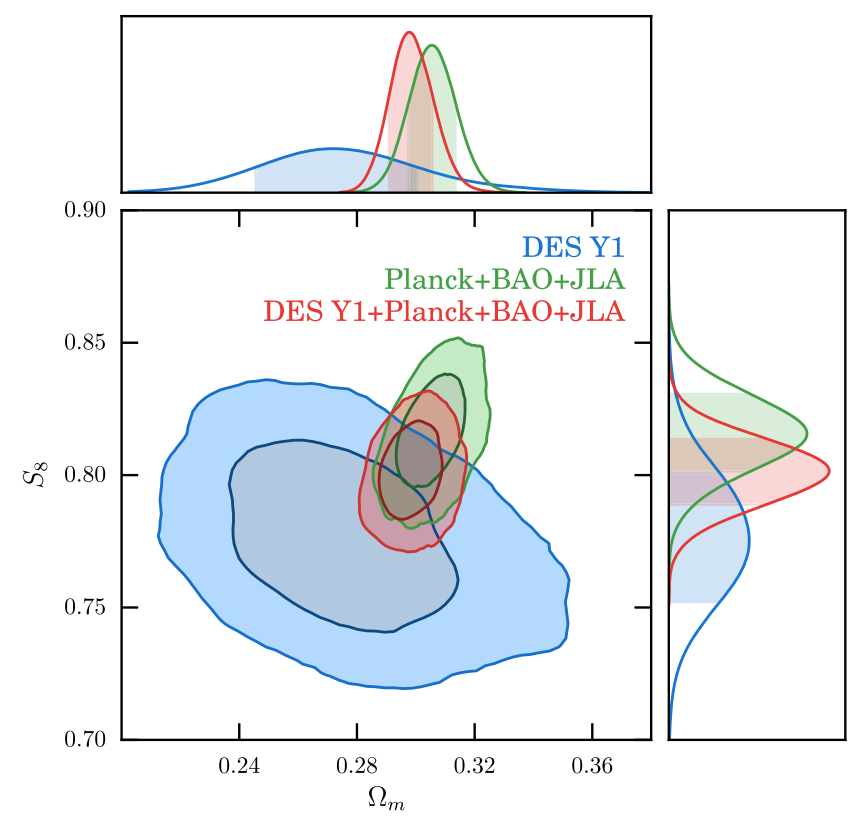

FIG. 13. $\Lambda \mathrm{CDM}$ constraints from all three two-point functions within DES and BAO, JLA, and Planck (with lensing) in the $\Omega_{m}-S_{8}$ plane.

another [53]. Here, "Planck" includes the data from the four-point function of the $\mathrm{CMB}$, which captures the effect of lensing due to large-scale structure at late times. Figure 13 shows the constraints in the $\Omega_{m}-S_{8}$ plane from this bundle of data sets and from DES Y1, in the $\Lambda$ CDM model. Here, the apparent consistency of the data sets is borne out by the Bayes factor for data set consistency [Eq. (5.3)]:

$$
\frac{P(\mathrm{JLA}+\mathrm{Planck}+\mathrm{BAO}+\mathrm{DES} \mathrm{Y} 1)}{P(\mathrm{JLA}+\mathrm{BAO}+\text { Planck }) P(\text { DES Y } 1)}=35 .
$$

Combining all of these leads to the tightest constraints yet on $\Lambda$ CDM parameters, shown in Table II. Highlighting some of these, at $68 \%$ C.L., the combination of DES with these external data sets yields

$$
\Omega_{m}=0.298 \pm 0.007 .
$$

This value is about $1 \sigma$ lower than the value without DES Y1, with comparable error bars. The clustering amplitude is also constrained at the percent level:

$$
\begin{aligned}
& \sigma_{8}=0.808_{-0.017}^{+0.009} \\
& S_{8}=0.802 \pm 0.012 .
\end{aligned}
$$

Note that fortuitously, because $\Omega_{m}$ is so close to 0.3 , the difference in the central values of $\sigma_{8}$ and $S_{8}$ is negligible. The combined result is about $1 \sigma$ lower than the inference without DES, and the constraints are tighter by about $20 \%$. 


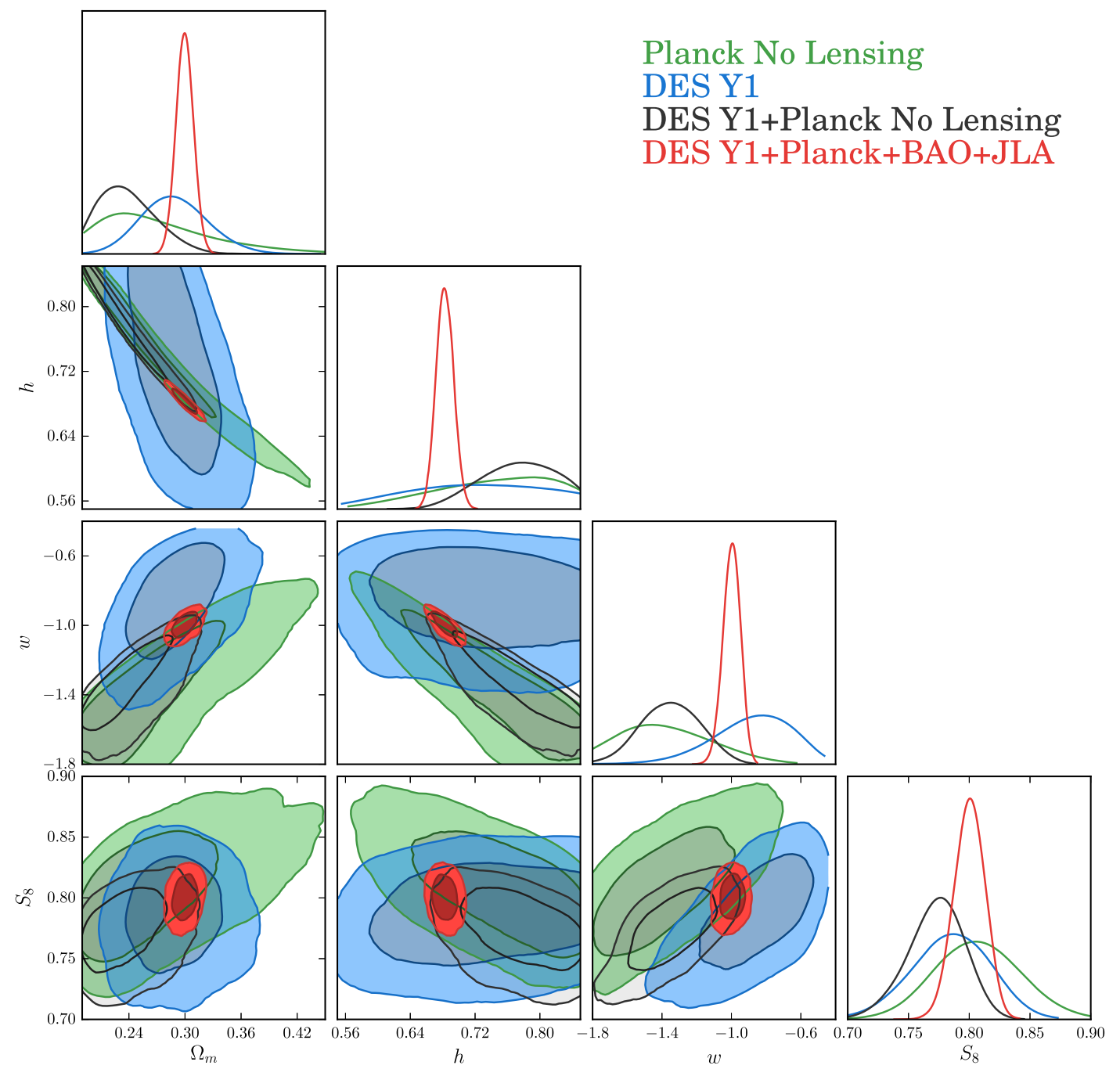

FIG. 14. $w$ CDM constraints from the three combined probes in DES Y1 and Planck with no lensing in the $\Omega_{m}-w-S_{8}-h$ subspace. Note the strong degeneracy between $h$ and $w$ from Planck data.

As mentioned above, the lower value of $\Omega_{m}$ leads to a higher value of the Hubble constant:

$h=0.658_{-0.027}^{+0.019} \quad$ (Planck: no lensing)

$h=0.685_{-0.007}^{+0.005} \quad(\mathrm{DES} \mathrm{Y} 1+\mathrm{JLA}+\mathrm{BAO}+$ Planck $)$

with neutrino mass varied.

\section{C. $w \mathrm{CDM}$}

Figure 14 shows the results in the extended $w C D M$ parameter space using Planck alone, DES alone, the two combined, and the two with the addition of $\mathrm{BAO}+\mathrm{SNe}$. As discussed in Ref. [53], the constraints on the dark energy equation of state from Planck alone are misleading. They stem from the measurement of the distance to the last scattering surface, and that distance (in a flat universe) depends upon the Hubble constant as well, so there is a strong $w-h$ degeneracy. The low values of $w$ seen in
Fig. 14 from Planck alone correspond to very large values of $h$. Since DES is not sensitive to the Hubble constant, it does not break this degeneracy. Additionally, the Bayes factor in Eq. (6.4) that quantifies whether adding the extra parameter $w$ is warranted is $R_{w}=0.7$. Therefore, opening up the dark energy equation of state is not favored on a formal level for the DES + Planck combination. Finally, the Bayes factor for combining DES and Planck (no lensing) in $w \mathrm{CDM}$ is equal to 10.3 , indicating "strong" evidence that the two data sets are consistent. DES Y1 and Planck jointly constrain the equation of state to $w=-1.35_{-0.17}^{+0.16}$, which is about $2 \sigma$ away from the cosmological-constant value.

The addition of $\mathrm{BAO}, \mathrm{SNe}$, and Planck lensing data to the DES + Planck combination yields the red contours in Fig. 14, shifting the solution substantially along the Planck degeneracy direction, demonstrating (i) the problems mentioned above with the DES + Planck (no lensing) combination and (ii) that these problems are resolved when other 
data sets that restrict the Hubble parameter to reasonable values are introduced. The Bayes factor for combination of Planck (no lensing) with the low- $z$ suite of DES + BAO + $\mathrm{SNe}$ in the $w \mathrm{CDM}$ model is $R=89$ substantially more supportive of the combination of experiments than the case for Planck and DES alone. The DES + Planck + BAO + SNe solution shows good consistency in the $\Omega_{m}-w-S_{8}$ subspace and yields our final constraint on the dark energy equation of state:

$$
w=-1.00_{-0.04}^{+0.05} \text {. }
$$

DES Y1 reduces the width of the allowed $68 \%$ region by $10 \%$. The evidence ratio $R_{w}=0.1$ for this full combination of data sets, disfavoring the introduction of $w$ as a free parameter.

\section{Neutrino mass}

The lower power observed in DES (relative to Planck) has implications for the constraint on the sum of the neutrino masses, as shown in Fig. 15. The current most stringent constraint comes from the cosmic microwave background and Lyman-alpha forest [149]. The experiments considered here (DES, JLA, and BAO) represent an independent set and so offer an alternative method for measuring the clustering of matter as a function of scale and redshift, which is one of the key drivers of the neutrino constraints. The 95\% C.L. upper limit on the sum of the neutrino masses in $\Lambda \mathrm{CDM}$ becomes less constraining:

$$
\sum m_{\nu}<0.26 \mathrm{eV}
$$

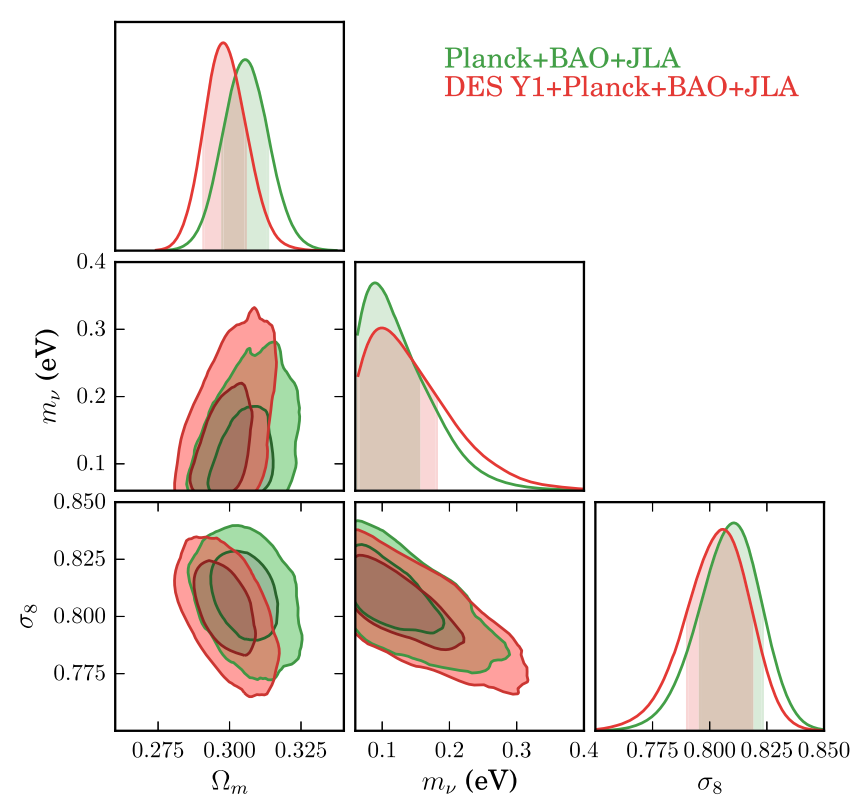

FIG. 15. $\Lambda$ CDM constraints on the sum of the neutrino masses from DES and other experiments. The lower power observed in DES can be accommodated either by lowering $\Omega$ or $\sigma_{8}$ or by increasing the sum of the neutrino masses.
Adding in DES Y1 loosens the constraint by close to $20 \%$ (from $0.22 \mathrm{eV}$ ). This is consistent with our finding that the clustering amplitude in DES Y1 is slightly lower than expected in $\Lambda \mathrm{CDM}$ informed by Planck. The three ways of reducing the clustering amplitude are to reduce $\Omega_{m}$, reduce $\sigma_{8}$, or increase the sum of the neutrino masses. The best-fit cosmology moves all three of these parameters slightly in the direction of less clustering in the present-day Universe.

We may, conversely, be concerned about the effect of priors on $\Omega_{\nu} h^{2}$ on the cosmological inferences in this paper. The results for DES Y1 and Planck depicted in Fig. 10 in $\Lambda \mathrm{CDM}$ were obtained when varying the sum of the neutrino masses. Neutrinos have mass [150], and the sum of the masses of the three light neutrinos is indeed unknown, so this parameter does need to be varied. However, many previous analyses have either set the sum to zero or to the minimum value allowed by oscillation experiments $\left(\sum m_{\nu}=0.06 \mathrm{eV}\right)$, so it is of interest to see if fixing neutrino mass alters any of our conclusions. In particular, does this alter the level of agreement between low- and highredshift probes in $\Lambda$ CDM? Figure 16 shows the extreme case of fixing the neutrino masses to the lowest value allowed by oscillation data: both the DES and Planck constraints in the $\Omega_{m}-S_{8}$ plane change. The Planck contours shrink toward the low $-\Omega_{m}$ side of their contours, while the DES constraints shift slightly to lower $\Omega_{m}$ and higher $S_{8}$. The Bayes factor for the combination of DES and Planck in the $\Lambda \mathrm{CDM}$ space changes from $R=6.6$ to $R=3.4$ when the minimal neutrino mass is enforced. DES and Planck therefore

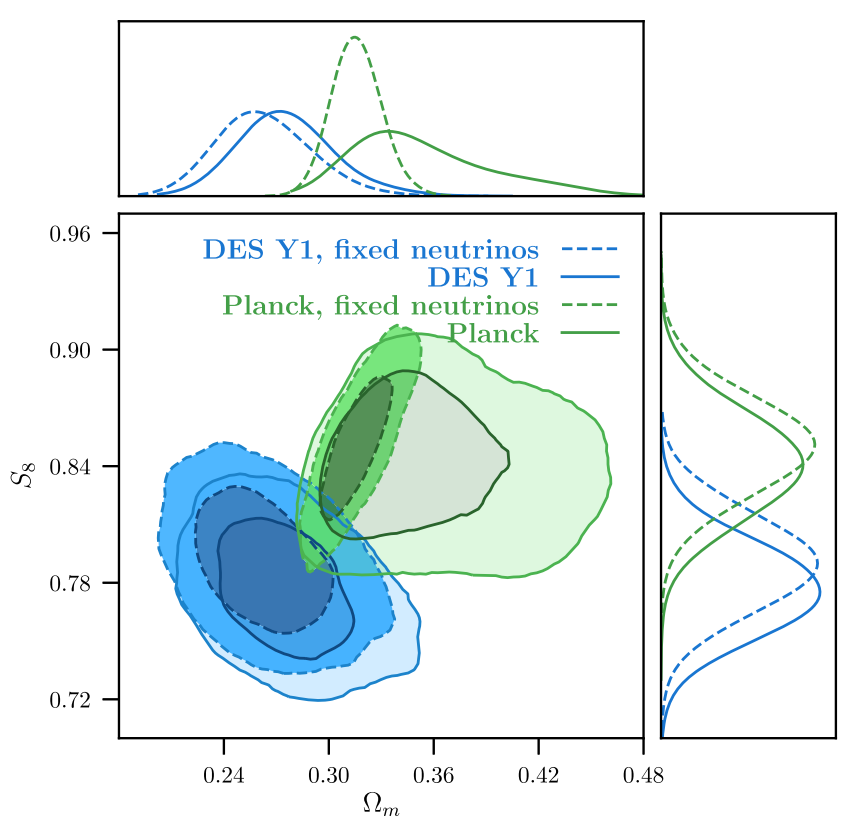

FIG. 16. $\Lambda$ CDM constraints on $\Omega_{m}$ and $\sigma_{8}$ from Planck without lensing and all three probes in DES. In contrast to all other plots in this paper, the dark contours here show the results when the sum of the neutrino masses was held fixed at its minimum allowed value of $0.06 \mathrm{eV}$. 
continue to agree, as seen in Fig. 16: when the neutrino mass is fixed, the area in the $\Omega_{m}-S_{8}$ plane allowed by Planck is much smaller than when $\Omega_{\nu} h^{2}$ varies, but there remains a substantial overlap between the Planck and DES contours.

Finally, fixing the neutrino mass allows us to compare directly to previous analyses that did the same. Although there are other differences in the analyses, such as the widths of the priors, treatments of systematics, and covariance matrix generation, fixing the neutrino mass facilitates a more accurate comparison. On the main parameter $S_{8}$ within $\Lambda \mathrm{CDM}$, again with neutrino mass fixed, the comparison is

$$
\begin{array}{rlr}
S_{8} & =0.793_{-0.026}^{+0.019} \quad \text { DES Y1 } \\
& =0.801 \pm 0.032 \quad \text { KiDS + GAMA [67] } \\
& =0.742 \pm 0.035 \quad \text { KiDS + 2dFLenS + BOSS [68], }
\end{array}
$$

so we agree with KiDS + GAMA, and differ from KiDS + $2 \mathrm{dFLenS}+$ BOSS by only about $1.2 \sigma$, indicating good statistical agreement.

\section{CONCLUSIONS}

We have presented cosmological results from a combined analysis of galaxy clustering and weak gravitational lensing, using imaging data from the first year of DES. These combined probes demonstrate that cosmic surveys using clustering measurements have now attained constraining power comparable to the cosmic microwave background in the $\Omega_{m}-S_{8}$ plane, heralding a new era in cosmology. The combined constraints on several cosmological parameters are the most precise to date.

The constraints on $\Omega_{m}$ from the CMB stem from the impact of the matter density on the relative heights of the acoustic peaks in the cosmic plasma when the Universe was only 380,000 years old and from the distance between us today and the CMB last scattering surface. The CMB constraints on $S_{8}$ are an expression of both the very small rms fluctuations in the density at that early time and the model's prediction for how rapidly they would grow over billions of years due to gravitational instability. The measurements themselves are of course in microwave bands and probed the Universe when it was extremely smooth. DES is different in every way: it probes in optical bands billions of years later when the Universe had evolved to be highly inhomogeneous. Instead of using the radiation as a tracer, DES uses galaxies and shear. It is truly extraordinary that a simple model makes consistent predictions for these vastly different sets of measurements.

The results presented here enable precise tests of the $\Lambda \mathrm{CDM}$ and $w \mathrm{CDM}$ models, as shown in Figs. 10 and 14. Our main findings are:

(i) DES Y1 constraints on $\Omega_{m}$ and $S_{8}$ in $\Lambda \mathrm{CDM}$ are competitive (in terms of their uncertainties) and compatible (according to tests of the Bayesian evidence) with constraints derived from Planck observations of the CMB. This is true even though the visual comparison (Fig. 10) of DES Y1 and Planck shows differences at the $1 \sigma$ to $2 \sigma$ level, in the direction of offsets that other recent lensing studies have reported.

(ii) The statistical consistency allows us to combine DES Y1 results with Planck, and, in addition, with BAO and supernova data sets. This yields $S_{8}=$ $0.802 \pm 0.012$ and $\Omega_{m}=0.298 \pm 0.007$ in $\Lambda \mathrm{CDM}$, the tightest such constraints to date (Fig. 13).

(iii) The $w$ CDM likelihoods from DES and Planck each constrain $w$ poorly; moreover, allowing $w$ as a free parameter maintains the consistency of the two data sets. DES is also consistent with the bundle of Planck, BAO, and supernova data, and this combination tightly constrains the equation-of-state parameter, $w=-1.00_{-0.04}^{+0.05}$ (Fig. 14).

(iv) The two-point functions measured in DES Y1 contain some information on two other open questions in cosmological physics: the combination of DES and Planck shifts the Planck constraints on the Hubble constant by more than $1 \sigma$ in the direction of local measurements (Fig. 12), and the joint constraints on neutrino mass slightly loosens the bound from external experiments to $\sum m_{\nu}<0.26 \mathrm{eV}$ (95\% C.L.) (Fig. 15).

(v) All results are based on redundant implementations and tests of the most critical components. They are robust to a comprehensive set of checks that we defined a priori and made while blind to the resulting cosmological parameters (see Sec. V and Appendix A). All related analyses, unless explicitly noted otherwise, marginalize over the relevant measurement systematics and neutrino mass.

(vi) Joint analyses of the three two-point functions of weak lensing and galaxy density fields have also been executed recently by the combination of the KiDS weak lensing data with the GAMA [67] and 2dfLenS [68] spectroscopic galaxy surveys, yielding $\Lambda \mathrm{CDM}$ bounds on $S_{8}$ that are in statistical agreement with ours; see Eq. (7.7). DES Y1 uncertainties are roughly $\sqrt{2}$ narrower than those from KiDS-450; while one might have expected a greater improvement considering the $\sim 3 \times$ increase in survey area, we caution against any detailed comparison of values or uncertainties until the analyses are homogenized to similar choices of scales, priors on neutrino masses, and treatments of observational systematic uncertainties.

The next round of cosmological analyses of DES data will include data from the first three years of the survey (DES Y3), which cover more than three times as much area to greater depth than Y1, and will incorporate constraints from clusters, supernovae, and cross-correlation with $\mathrm{CMB}$ lensing, shedding more light on dark energy and cosmic acceleration. 


\section{ACKNOWLEDGMENTS}

Funding for the DES Projects has been provided by the U.S. Department of Energy, the U.S. National Science Foundation, the Ministry of Science and Education of Spain, the Science and Technology Facilities Council of the United Kingdom, the Higher Education Funding Council for England, the National Center for Supercomputing Applications at the University of Illinois at UrbanaChampaign, the Kavli Institute of Cosmological Physics at the University of Chicago, the Center for Cosmology and Astro-Particle Physics at Ohio State University, the Mitchell Institute for Fundamental Physics and Astronomy at Texas A\&M University, Financiadora de Estudos e Projetos, Fundação Carlos Chagas Filho de Amparo à Pesquisa do Estado do Rio de Janeiro, Conselho Nacional de Desenvolvimento Científico e Tecnológico and the Ministério da Ciência, Tecnologia e Inovação, the Deutsche Forschungsgemeinschaft, and the collaborating institutions in the Dark Energy Survey. The Collaborating Institutions are Argonne National Laboratory, the University of California at Santa Cruz, the University of Cambridge, Centro de Investigaciones Energéticas, Medioambientales y Tecnológicas-Madrid, the University of Chicago, University College London, the DES-Brazil Consortium, the University of Edinburgh, the Eidgenössische Technische Hochschule Zürich, Fermi National Accelerator Laboratory, the University of Illinois at Urbana-Champaign, the Institut de Ciències de l'Espai, the Institut de Física d'Altes Energies, Lawrence Berkeley National Laboratory, the Ludwig-Maximilians Universität München and the associated Excellence Cluster Universe, the University of Michigan, the National Optical Astronomy Observatory, the University of Nottingham, the Ohio State University, the University of Pennsylvania, the University of Portsmouth, SLAC National Accelerator Laboratory, Stanford University, the University of Sussex, Texas A\&M University, and the OzDES Membership Consortium. This work was based in part on observations at Cerro Tololo Inter-American Observatory, National Optical Astronomy Observatory, which is operated by the Association of Universities for Research in Astronomy under a cooperative agreement with the National Science Foundation. The DES data management system is supported by the National Science Foundation under Grants No. AST-1138766 and No. AST-1536171. The DES participants from Spanish institutions are partially supported by MINECO under Grants No. AYA2015-71825, No. ESP2015-88861, No. FPA2015-68048, No. SEV-2012-0234, No. SEV2016-0597, and No. MDM-2015-0509, some of which include European Research Development Fund (ERDF) funds from the European Union. I. F. A.E. is partially funded by the Centres de Recerce de Catalunya (CERCA) program of the Generalitat de Catalunya. Research leading to these results has received funding from the European
Research Council under the European Union's Seventh Framework Program (FP7/2007-2013) including European Research Council Grants No. 240672, No. 291329, and No. 306478. We acknowledge support from the Australian Research Council Centre of Excellence for All-sky Astrophysics, through Project No. CE110001020. This manuscript has been authored by Fermi Research Alliance, LLC, under Contract No. DE-AC0207CH11359 with the U.S. Department of Energy, Office of Science, Office of High Energy Physics. The United States Government retains and the publisher, by accepting the article for publication, acknowledges that the United States Government retains a nonexclusive, paid-up, irrevocable, worldwide license to publish or reproduce the published form of this manuscript, or allow others to do so, for United States Government purposes. We are grateful to Anže Slosar for helpful communications. Many of the figures in this paper were produced with CHAINCONSUMER [151]. This research used resources of the National Energy Research Scientific Computing Center, a DOE Office of Science User Facility supported by the Office of Science of the U.S. Department of Energy under Contract No. DEAC02-05CH11231.

\section{APPENDIX A: UNBLINDING TESTS}

Here, we describe some of the results of the tests enumerated in Sec. V. The most relevant metrics are the

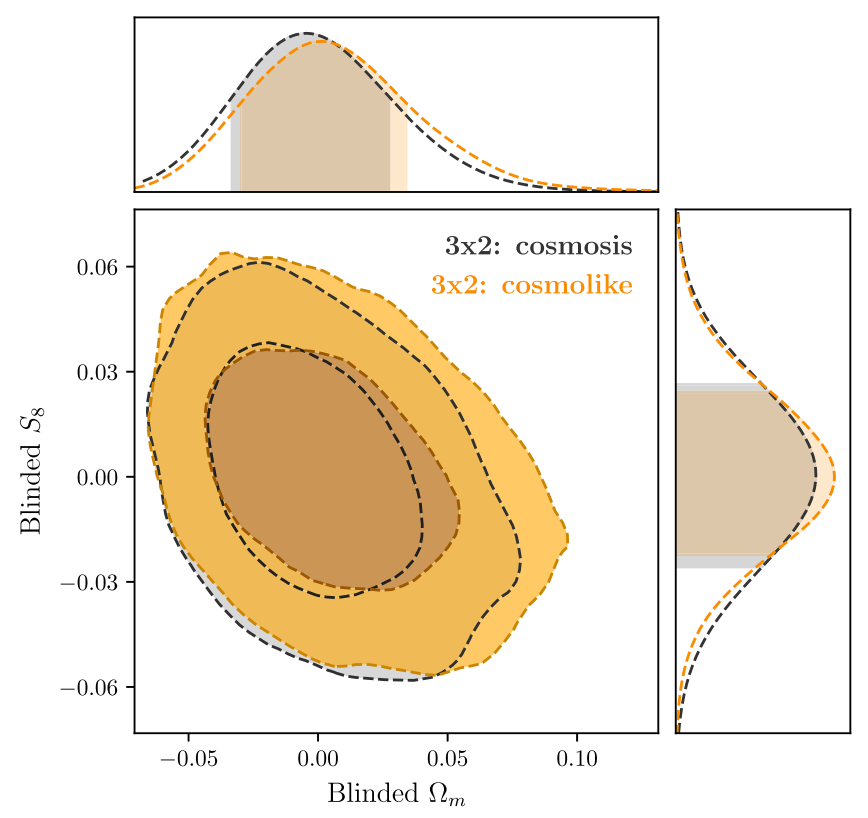

FIG. 17. Blinded constraints on $\Omega_{m}$ and $S_{8}$ from all three twopoint functions in DES Y1 using two separate analysis pipelines on the data. Both contours are shifted by the means of the posteriors obtained from CosmoSIS, so that the CosmoLike contours could in principle be centered away from the origin. This figure was made prior to unblinding, thus without the update to the covariance described in Appendix C. 


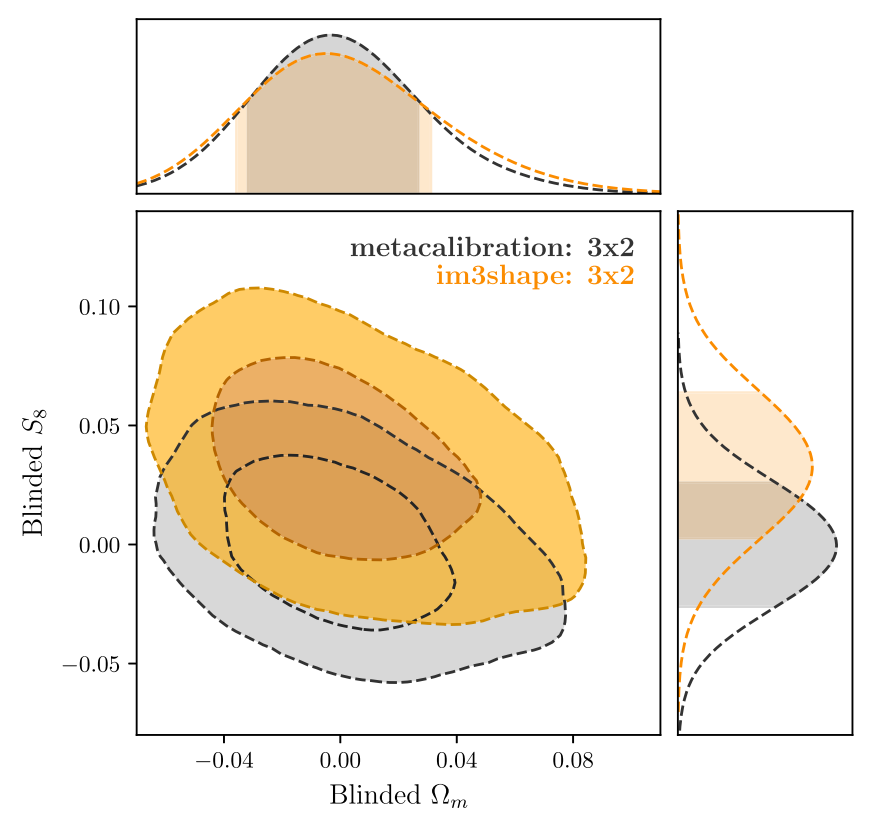

FIG. 18. Blinded constraints from DES Y1 on $\Omega_{m}$ and $S_{8}$ from all three combined probes, using the two independent shape pipelines METACALIBRATION and IM3SHAPE.

values of the cosmological parameters best constrained by DES Y1, namely $\Omega_{m}$ and $S_{8}$. We report here on the few instances in which the robustness tests yielded shifts in either the values or the uncertainties on $S_{8}$ or $\Omega_{m}$ exceeding $10 \%$ of their $68 \%$ C.L. intervals.
Figure 17 shows the result of test 7. As CosmoSIS and COSMOLIKE use the same data and models, there should in principle be no difference between them except for the sampling noise of their finite MCMC chains. CosmoSIS yields error bars on $\Omega_{m}$ slightly smaller than those obtained from CosmoLiKe, with a $<0.2 \sigma$ change in central value. The $S_{8}$ constraints agree to better than a percent, and the error bars agree to within 3\%. These numbers and the contours shown in Fig. 17 improved over the results obtained before unblinding, when the difference in the error bars was larger. Longer EMCEE chains account for the improvement, so it is conceivable that these small differences-which do not affect our conclusions-go away with even longer chains.

When carrying out test 7 , we found that for both METACALIBRATION and IM3SHAPE, almost all of the parameters were tightly constrained to lie well within their sampling ranges. The lone exception was the power law of the intrinsic-alignment signal, $\eta_{\mathrm{IA}}$, which had an error that is large relative to the prior, but this was entirely expected, as our simulations indicated that the Y1 data have little constraining power on $\eta_{\mathrm{IA}}$. For those parameters with more informative priors, the posteriors typically fell close to the priors, indicating that the data were consistent with the calibrations described in Refs. [87,88]. One exception was the IM3SHAPE value $\Delta z_{s}^{4}$, the shift in the mean value of the redshift in the fourth source bin, where the posterior and prior differed by close to $1 \sigma$.
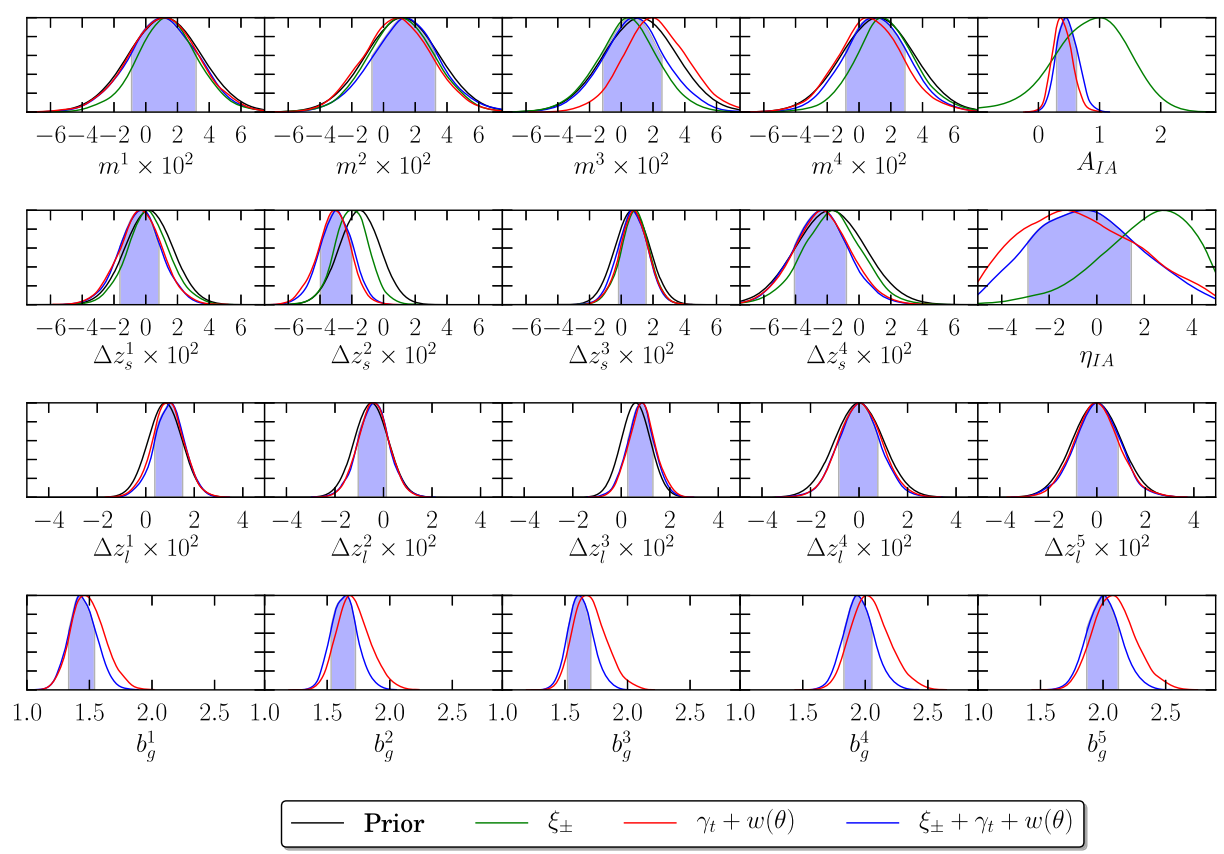

FIG. 19. The posteriors from cosmic shear, from $w(\theta)+\gamma_{t}(\theta)$, and for all three probes using the METACALIBRATION pipeline for all 20 nuisance parameters used in the $\Lambda \mathrm{CDM}$ analysis. The priors are also shown. There are no priors for the bias and intrinsic-alignment parameters, and the biases and the lens shifts are not constrained by $\xi_{ \pm}$. Therefore, the bottom panels have only two curves: posteriors from $w(\theta)+\gamma_{t}(\theta)$ and from all three probes. Similarly, there are only three curves for the two intrinsic alignment parameters. 
We next compare the METACALIBRATION and IM3SHAPE constraints in the $\Omega_{m}-S_{*}$ plane, noting that Fig. 12 of Ref. [92] already shows good agreement between the two pipelines on inferences purely with cosmic shear. Figure 18 shows that when all $3 \times 2$-point data are combined, METACALIBRATION and IM3SHAPE are in good agreement. Note also that their corresponding data vectors are not directly comparable, since they bin and weight the source galaxies differently and thus have distinct redshift distributions - they can be properly compared only in cosmological-parameter tests such as this.

For test 9, we deleted from the data vector angular scales $<20$ arc minutes from $\xi_{+},<150$ arc minutes from $\xi_{-}$, $<65$ arc minutes from $\gamma_{t}$, and $<50$ arc minutes from $w(\theta)$. The cosmological parameter constraints expanded slightly, as expected, but shifted by much less than $1 \sigma$.

Finally, although we looked at these blinded, Fig. 19 shows the posteriors of all 20 nuisance parameters used to model the data. Note the agreement of the two sets of probes with each other and with the priors on the parameters.

Before unblinding, we listed several additional robustness tests that would be carried out after unblinding. These are described in Appendix B.

\section{APPENDIX B: ROBUSTNESS OF RESULTS}

Here, we test the impact on the final results of some of the choices made during analysis. These tests, conducted while unblinded but identified beforehand, supplement those described in Sec. V.

All of our inferences require assumptions about the redshift distributions for the source and lens galaxies. We have quantified the uncertainties in the redshift distributions with a shift parameter, as described in and around Eq. (2.1). This allows for the means of the distributions to change but does not allow for any flexibility in the shapes. We now check that the uncertainty in the photometric distributions in the source bins is adequately captured by using the BPZ redshift distribution accompanied by the free shift parameter in each bin. Instead of redshift distributions obtained via BPZ, we use those obtained directly from the COSMOS data, as described in Ref. [88]. As shown in Fig. 4 there, the shapes of the redshift distributions are quite different from one another, so if we obtain the same cosmological results using these different shape $n(z)$ 's, we will have demonstrated that the detailed shapes do not drive the constraints. Again, we allow for a free shift in each of the source distributions. Figure 20 shows that the ensuing constraints are virtually identical to those that use the BPZ $n(z)$ 's for the source galaxies, suggesting that our results are indeed sensitive only to the means of the redshift distributions in each bin, and not to the detailed shapes.

We also considered the impact of the choices made while computing the covariance matrix. These choices require assumptions about all 26 parameters that are varied. We

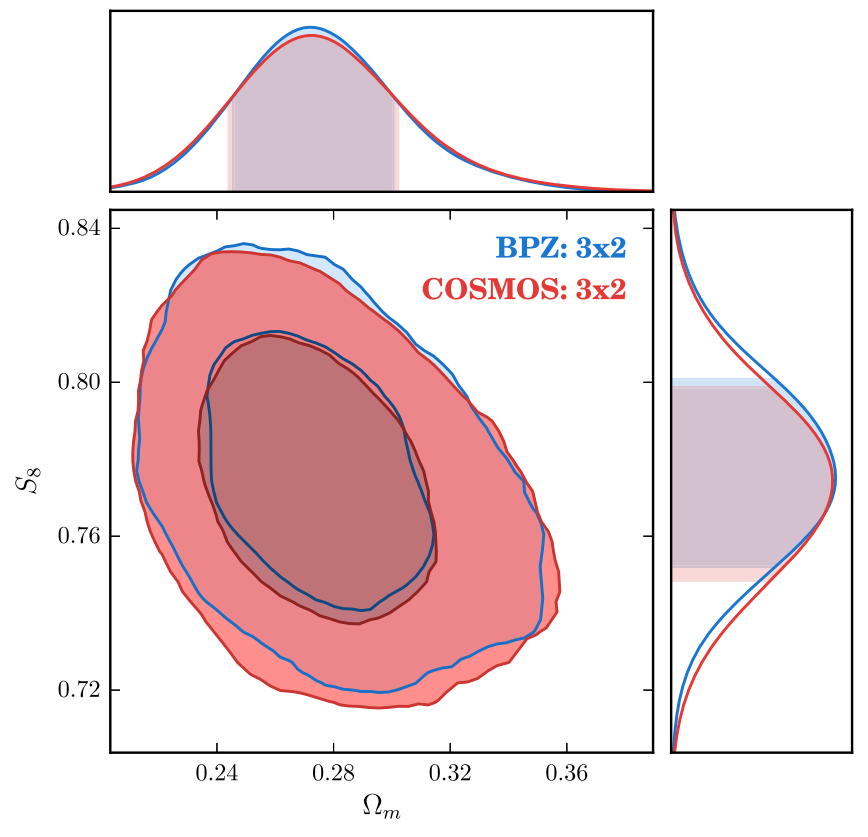

FIG. 20. Constraints on $\Omega_{m}$ and $S_{8}$ when using the shifted BPZ redshift distributions as the default for $n_{s}^{i}(z)$, compared with those obtained when using the COSMOS redshift distribution, which have different shape, as seen in Fig. 4 of Ref. [88].

generated an initial covariance matrix assuming fiducial values for these parameters but then after unblinding recomputed it using the means of the posteriors of all the parameters as input. How much did this (small) change in the covariance matrix affect our final results? Figure 21

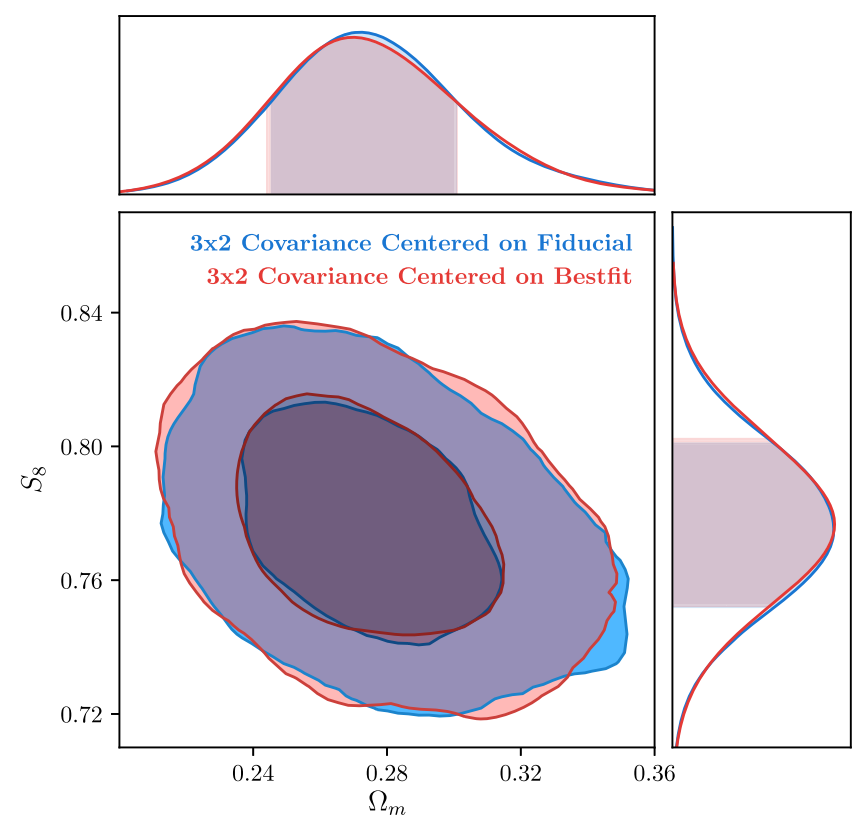

FIG. 21. Constraints on $\Omega_{m}$ and $S_{8}$ using the fiducial covariance matrix and using the covariance based on the cosmological model centered on the means of the posteriors ("Bestfit") obtained after unblinding. The two agree very well, indicating little dependence on the fiducial model assumed for the covariance. 


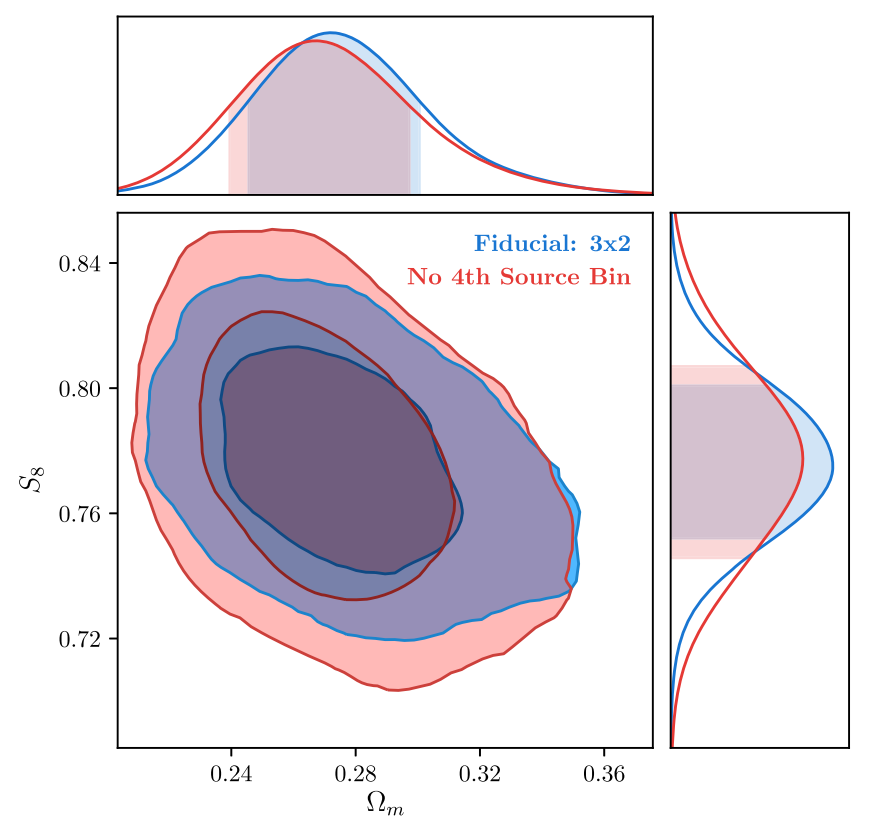

FIG. 22. Constraints from all three probes using all four source bins ("Fiducial") and with the fourth source bin removed.

shows that the updated covariance matrix had essentially no impact on our final parameter determination.

There are no REDMAGIC galaxies in our catalog at redshifts overlapping the fourth source bin, so the only way to verify the mean redshift of the galaxies in that bin is to use the COSMOS galaxies. All the other source bins benefit from the twofold validation scheme. We therefore checked to see if removing the highest redshift bin affected our constraints. Figure 22 shows that our fiducial constraints are completely consistent with the looser ones obtained when the highest redshift bin is removed.

\section{APPENDIX C: CHANGES TO FIDUCIAL COVARIANCE}

In the first public version of this paper, the value we reported for $\chi^{2}$ between our fiducial data vector and our best-fit model was $\chi^{2}=572$. This has to be compared to the degrees of freedom of our fit; note that $N_{\text {dof }}=N_{\text {data }}-$ $N_{\text {param }}$ is not entirely applicable in our situation since we have strong priors on several of our parameters. We account for this by assuming an effective number of parameters $N_{\text {param,eff }}=13$, which is the number of parameters that are not tightly constrained by our priors. This resulted in $N_{\text {dof,eff }} \approx 457-13=444$. The reduced $\chi^{2}$ in the first version of the analysis was hence $\approx 1.29$ which, while clearly high, was below the threshold of 1.4 which had been set as a requirement before unblinding the analysis.

Following referee comments to the first version of this paper, we were fortunately able track down the cause of this elevated $\chi^{2}$ to two inaccuracies of our model covariance:

(1) We had analytically calculated the number of galaxy pairs falling into a particular angular bin using simple geometric approximations. These approximations can fail for several reasons. First, the finite size of our footprint leads to a decrease in the observed density of galaxy pairs found on scales comparable to the footprint diameter. Second, the mask pattern on scales smaller than the angular scales used in our data vector decreases the number of pairs found in each angular bin by a factor that is almost uniform across angular scales. And, third, the clustering of galaxies increases the number of galaxy pairs found on small scales.

(2) When estimating the dispersion of intrinsic galaxy shapes $\sigma_{\epsilon}$, we measured the variance of the galaxy shape within our entire source sample. This ignored propagating the effect of significant differences in shape dispersion between different source redshift bins to the covariance matrix.

Both of these analysis improvements affect the noise contribution (shot noise and shape noise) to the diagonal of our covariance matrix. With these changes identified, we recomputed the shape-noise and shot-noise terms using the actual numbers of galaxy pairs found in the data and estimating the shape dispersion separately for each source redshift bin. In addition, we recomputed the cosmic variance terms in the covariance using our best-fit cosmology.

These changes in our covariance improve the value of $\chi^{2}$ obtained for our best-fit model in the $3 \times 2 \Lambda \mathrm{CDM}$ analysis to $497\left(\chi^{2} /\right.$ dof $\left.\approx 1.12\right)$. Note that a change in covariance affects both the width and the location of parameter constraints, but both of these changed very little after the covariance update; by far, the dominant effect was the improvement in overall $\chi^{2}$. As an added bonus, the two shear pipelines, METACALIBRATION and IM3SHAPE, are now in a better mutual agreement than before (see Fig. 18).
[1] A. G. Riess et al., Astron. J. 116, 1009 (1998).

[2] S. Perlmutter et al. .(Supernova Cosmology Project Collaboration), Astrophys. J. 517, 565 (1999).
[3] A. Einstein, Sitzungsber. Preuss. Akad. Wiss. Berlin (Math. Phys.) 1917, 142 (1917).

[4] O. Lahav and A. R. Liddle, arXiv:1401.1389. 
[5] D. H. Weinberg, M. J. Mortonson, D. J. Eisenstein, C. Hirata, A. G. Riess, and E. Rozo, Phys. Rep. 530, 87 (2013).

[6] J. Frieman, M. Turner, and D. Huterer, Annu. Rev. Astron. Astrophys. 46, 385 (2008).

[7] DES Collaboration, arXiv:astro-ph/0510346.

[8] G. R. Blumenthal, S. M. Faber, J. R. Primack, and M. J. Rees, Nature (London) 311, 517 (1984).

[9] S. J. Maddox, G. Efstathiou, W. J. Sutherland, and J. Loveday, Mon. Not. R. Astron. Soc. 243, 692 (1990).

[10] C. M. Baugh, Mon. Not. R. Astron. Soc. 280, 267 (1996).

[11] S. J. Maddox, G. Efstathiou, and W. J. Sutherland, Mon. Not. R. Astron. Soc. 283, 1227 (1996).

[12] D. J. Eisenstein and M. Zaldarriaga, Astrophys. J. 546, 2 (2001).

[13] C. A. Collins, R. C. Nichol, and S. L. Lumsden, Mon. Not. R. Astron. Soc. 254, 295 (1992).

[14] I. Szapudi and E. Gaztanaga, Mon. Not. R. Astron. Soc. 300, 493 (1998).

[15] D. Huterer, L. Knox, and R. C. Nichol, Astrophys. J. 555, 547 (2001).

[16] W. Saunders et al., Mon. Not. R. Astron. Soc. 317, 55 (2000).

[17] A. J. S. Hamilton and M. Tegmark, Mon. Not. R. Astron. Soc. 330, 506 (2002).

[18] S. Cole et al. (2dFGRS Collaboration), Mon. Not. R. Astron. Soc. 362, 505 (2005).

[19] M. Tegmark et al. (SDSS Collaboration), Phys. Rev. D 74, 123507 (2006).

[20] N. Kaiser, Astrophys. J. 284, L9 (1984).

[21] J. A. Tyson, R. A. Wenk, and F. Valdes, Astrophys. J. 349, L1 (1990).

[22] T. G. Brainerd, R. D. Blandford, and I. Smail, Astrophys. J. 466, 623 (1996).

[23] D. J. Bacon, A. R. Refregier, and R. S. Ellis, Mon. Not. R. Astron. Soc. 318, 625 (2000).

[24] N. Kaiser, G. Wilson, and G. A. Luppino, arXiv:astro-ph/ 0003338.

[25] L. van Waerbeke et al., Astron. Astrophys. 358, 30 (2000).

[26] D. M. Wittman, J. A. Tyson, D. Kirkman, I. Dell'Antonio, and G. Bernstein, Nature (London) 405, 143 (2000).

[27] M. Jarvis, B. Jain, G. Bernstein, and D. Dolney, Astrophys. J. 644, 71 (2006).

[28] R. Massey et al., Astrophys. J. Suppl. Ser. 172, 239 (2007).

[29] T. Schrabback et al., Astron. Astrophys. 516, A63 (2010).

[30] H. Lin, S. Dodelson, H.-J. Seo, M. Soares-Santos, J. Annis, J. Hao, D. Johnston, J. M. Kubo, R. R. R. Reis, and M. Simet (SDSS Collaboration), Astrophys. J. 761, 15 (2012).

[31] C. Heymans et al., Mon. Not. R. Astron. Soc. 432, 2433 (2013).

[32] E. M. Huff, T. Eifler, C. M. Hirata, R. Mandelbaum, D. Schlegel, and U. Seljak, Mon. Not. R. Astron. Soc. 440, 1322 (2014).

[33] M. Kilbinger et al., Mon. Not. R. Astron. Soc. 430, 2200 (2013).

[34] M. J. Jee, J. A. Tyson, S. Hilbert, M. D. Schneider, S. Schmidt, and D. Wittman, Astrophys. J. 824, 77 (2016).

[35] H. Hildebrandt et al., Mon. Not. R. Astron. Soc. 465, 1454 (2017).
[36] S. Joudaki, C. Blake, C. Heymans, A. Choi, J. HarnoisDeraps, H. Hildebrandt, B. Joachimi, A. Johnson, A. Mead, D. Parkinson, M. Viola, and L. van Waerbeke, Mon. Not. R. Astron. Soc. 465, 2033 (2017).

[37] P. Fischer et al. (SDSS Collaboration), Astron. J. 120, 1198 (2000).

[38] E. S. Sheldon et al. (SDSS Collaboration), Astron. J. 127, 2544 (2004).

[39] A. Leauthaud et al., Astrophys. J. 744, 159 (2012).

[40] R. Mandelbaum, U. Seljak, G. Kauffmann, C. M. Hirata, and J. Brinkmann, Mon. Not. R. Astron. Soc. 368, 715 (2006).

[41] D. E. Johnston, E. S. Sheldon, R. H. Wechsler, E. Rozo, B. P. Koester, J. A. Frieman, T. A. McKay, A. E. Evrard, M. R. Becker, and J. Annis (SDSS Collaboration), arXiv:0709.1159.

[42] M. Cacciato, F. C. v. d. Bosch, S. More, R. Li, H. J. Mo, and X. Yang, Mon. Not. R. Astron. Soc. 394, 929 (2009).

[43] R. Mandelbaum, A. Slosar, T. Baldauf, U. Seljak, C. M. Hirata, R. Nakajima, R. Reyes, and R. E. Smith, Mon. Not. R. Astron. Soc. 432, 1544 (2013).

[44] A. Choi, J. A. Tyson, C. B. Morrison, M. J. Jee, S. J. Schmidt, V. E. Margoniner, and D. M. Wittman, Astrophys. J. 759, 101 (2012).

[45] M. Velander et al., Mon. Not. R. Astron. Soc. 437, 2111 (2014).

[46] J. Clampitt et al. (DES Collaboration), Mon. Not. R. Astron. Soc. 465, 4204 (2017).

[47] A. Leauthaud et al., Mon. Not. R. Astron. Soc. 467, 3024 (2017).

[48] J. Kwan et al., Mon. Not. R. Astron. Soc. 464, 4045 (2017).

[49] S. W. Allen, A. E. Evrard, and A. B. Mantz, Annu. Rev. Astron. Astrophys. 49, 409 (2011).

[50] S. Alam et al. (BOSS Collaboration), Mon. Not. R. Astron. Soc. 470, 2617 (2017).

[51] G. Hinshaw et al. (WMAP Collaboration), Astrophys. J. Suppl. Ser. 208, 19 (2013).

[52] P. A. R. Ade et al. (Planck Collaboration), Astron. Astrophys. 571, A16 (2014).

[53] P. A. R. Ade et al. (Planck Collaboration), Astron. Astrophys. 594, A13 (2016).

[54] E. Calabrese et al., Phys. Rev. D 95, 063525 (2017).

[55] S. Das et al., Phys. Rev. Lett. 107, 021301 (2011).

[56] A. van Engelen et al., Astrophys. J. 756, 142 (2012).

[57] P. A. R. Ade et al. (Planck Collaboration), Astron. Astrophys. 594, A15 (2016).

[58] W. Hu and B. Jain, Phys. Rev. D 70, 043009 (2004).

[59] G. M. Bernstein, Astrophys. J. 695, 652 (2009).

[60] B. Joachimi and S. L. Bridle, Astron. Astrophys. 523, A1 (2010).

[61] A. Nicola, A. Refregier, and A. Amara, Phys. Rev. D 94, 083517 (2016).

[62] D. Huterer, M. Takada, G. Bernstein, and B. Jain, Mon. Not. R. Astron. Soc. 366, 101 (2006).

[63] P. Zhang, U.-L. Pen, and G. Bernstein, Mon. Not. R. Astron. Soc. 405, 359 (2010).

[64] Y. Park et al. (DES Collaboration), Phys. Rev. D 94, 063533 (2016). 
[65] S. Samuroff, M. Troxel, S. Bridle, J. Zuntz, N. MacCrann, E. Krause, T. Eifler, and D. Kirk, Mon. Not. R. Astron. Soc. 465, L20 (2017).

[66] P. Zhang, Astrophys. J. 720, 1090 (2010).

[67] E. van Uitert et al., arXiv:1706.05004.

[68] S. Joudaki et al., Mon. Not. R. Astron. Soc. 474, 4894 (2018).

[69] P. Melchior et al. (DES Collaboration), Mon. Not. R. Astron. Soc. 449, 2219 (2015).

[70] V. Vikram et al. (DES Collaboration), Phys. Rev. D 92, 022006 (2015).

[71] C. Chang et al. (DES Collaboration), Phys. Rev. Lett. 115, 051301 (2015).

[72] M. R. Becker et al. (DES Collaboration), Phys. Rev. D 94, 022002 (2016).

[73] DES Collaboration, Phys. Rev. D 94, 022001 (2016).

[74] M. Crocce et al. (DES Collaboration), Mon. Not. R. Astron. Soc. 455, 4301 (2016).

[75] D. Gruen et al. (DES Collaboration), Mon. Not. R. Astron. Soc. 455, 3367 (2016).

[76] N. MacCrann et al. (DES Collaboration), Mon. Not. R. Astron. Soc. 465, 2567 (2017).

[77] J. Prat et al. (DES Collaboration), Mon. Not. R. Astron. Soc. 473, 1667 (2018).

[78] L. Clerkin et al. (DES Collaboration), Mon. Not. R. Astron. Soc. 466, 1444 (2017).

[79] C. Chang et al. (DES Collaboration), Mon. Not. R. Astron. Soc. 459, 3203 (2016).

[80] P. Melchior et al. (DES Collaboration), Mon. Not. R. Astron. Soc. 469, 4899 (2017).

[81] T. Kacprzak et al. (DES Collaboration), Mon. Not. R. Astron. Soc. 463, 3653 (2016).

[82] C. Sanchez et al. (DES Collaboration), Mon. Not. R. Astron. Soc. 465, 746 (2017).

[83] T. Abbott (DES Collaboration), Mon. Not. R. Astron. Soc. 460, 1270 (2016).

[84] E. Krause et al. (DES Collaboration), arXiv:1706.09359 [Phys. Rev. D (to be published)].

[85] N. MacCrann et al. (DES Collaboration), arXiv: 1803.09795.

[86] A. Drlica-Wagner et al. (DES Collaboration), Astrophys. J. Suppl. Ser. (to be published).

[87] J. Zuntz et al. (DES Collaboration), Mon. Not. R. Astron. Soc. (to be published).

[88] B. Hoyle et al. (DES Collaboration), Mon. Not. R. Astron. Soc. (to be published).

[89] M. Gatti et al. (DES Collaboration), Mon. Not. R. Astron. Soc. 477, 1664 (2018).

[90] R. Cawthon et al. (DES Collaboration), Mon. Not. Roy. Astron. Soc. (to be published).

[91] C. Davis et al. (DES Collaboration), Mon. Not. R. Astron. Soc. (to be published).

[92] M. A. Troxel et al. (DES Collaboration), arXiv: 1708.01538 .

[93] J. Prat et al. (DES Collaboration), arXiv:1708.01537.

[94] J. Elvin-Poole et al. (DES Collaboration), arXiv: 1708.01536.

[95] B. Flaugher et al., Astron. J. 150, 150 (2015).

[96] S. Desai et al., Astrophys. J. 757, 83 (2012).
[97] I. Sevilla et al. (DES Collaboration), in Proceedings of the DPF-2011 Conference, Proceedings of the DPF-2011 Conference Providence, Rhode Island (2011).

[98] J. J. Mohr et al. (DES Collaboration), Proc. SPIE Int. Soc. Opt. Eng. 7016, OL (2008).

[99] E. Morganson et al. (DES Collaboration), Publ. Astron. Soc. Pac. 130, 074501 (2018).

[100] E. Rozo et al. (DES Collaboration), Mon. Not. R. Astron. Soc. 461, 1431 (2016).

[101] E. Huff and R. Mandelbaum, arXiv:1702.02600.

[102] E. S. Sheldon and E. M. Huff, Astrophys. J. 841, 24 (2017).

[103] J. Zuntz, T. Kacprzak, L. Voigt, M. Hirsch, B. Rowe, and S. Bridle, Mon. Not. R. Astron. Soc. 434, 1604 (2013).

[104] S. Samuroff et al. (DES Collaboration), Mon. Not. R. Astron. Soc. 475, 4524 (2018).

[105] D. Coe, N. Benítez, S. F. Sánchez, M. Jee, R. Bouwens, and H. Ford, Astron. J. 132, 926 (2006).

[106] C. Laigle et al., Astrophys. J. Suppl. Ser. 224, 24 (2016).

[107] M. Jarvis, G. Bernstein, and B. Jain, Mon. Not. R. Astron. Soc. 352, 338 (2004).

[108] M. A. Troxel and M. Ishak, Phys. Rep. 558, 1 (2015).

[109] J. Miralda-Escude, Astrophys. J. 380, 1 (1991).

[110] N. Kaiser, Astrophys. J. 388, 272 (1992).

[111] N. Kaiser, Astrophys. J. 498, 26 (1998).

[112] M. Kamionkowski, A. Babul, C. M. Cress, and A. Refregier, Mon. Not. R. Astron. Soc. 301, 1064 (1998).

[113] L. Hui, Astrophys. J. 519, L9 (1999).

[114] M. Bartelmann and P. Schneider, Phys. Rep. 340, 291 (2001).

[115] A. Refregier, Annu. Rev. Astron. Astrophys. 41, 645 (2003).

[116] H. Hoekstra and B. Jain, Annu. Rev. Nucl. Part. Sci. 58, 99 (2008).

[117] D. N. Limber, Astrophys. J. 119, 655 (1954).

[118] N. Kaiser, Astrophys. J. 388, 272 (1992).

[119] M. LoVerde and N. Afshordi, Phys. Rev. D 78, 123506 (2008).

[120] M. Kilbinger et al., Mon. Not. R. Astron. Soc. 472, 2126 (2017).

[121] A. Lewis, A. Challinor, and A. Lasenby, Astrophys. J. 538, 473 (2000).

[122] J. Lesgourgues, arXiv:1104.2932.

[123] R. E. Smith, J. A. Peacock, A. Jenkins, S. D. M. White, C. S. Frenk, F. R. Pearce, P. A. Thomas, G. Efstathiou, and H. M. P. Couchman, Mon. Not. R. Astron. Soc. 341, 1311 (2003).

[124] R. Takahashi, M. Sato, T. Nishimichi, A. Taruya, and M. Oguri, Astrophys. J. 761, 152 (2012).

[125] S. Bird, M. Viel, and M. G. Haehnelt, Mon. Not. R. Astron. Soc. 420, 2551 (2012).

[126] F. Villaescusa-Navarro, F. Marulli, M. Viel, E. Branchini, E. Castorina, E. Sefusatti, and S. Saito, J. Cosmol. Astropart. Phys. 03 (2014) 011.

[127] M. Biagetti, V. Desjacques, A. Kehagias, and A. Riotto, Phys. Rev. D 90, 045022 (2014).

[128] M. LoVerde, Phys. Rev. D 90, 083530 (2014).

[129] C. Patrignani et al. (Particle Data Group Collaboration), Chin. Phys. C 40, 100001 (2016).

[130] A. G. Riess et al., Astrophys. J. 826, 56 (2016). 
[131] E. Krause and T. Eifler, Mon. Not. R. Astron. Soc. 470, 2100 (2017).

[132] T. Eifler, P. Schneider, and J. Hartlap, Astron. Astrophys. 502, 721 (2009).

[133] J. Zuntz, M. Paterno, E. Jennings, D. Rudd, A. Manzotti, S. Dodelson, S. Bridle, S. Sehrish, and J. Kowalkowski, Astron. Comput. 12, 45 (2015).

[134] F. Feroz, M. P. Hobson, and M. Bridges, Mon. Not. R. Astron. Soc. 398, 1601 (2009).

[135] D. Foreman-Mackey, D. W. Hogg, D. Lang, and J. Goodman, Publ. Astron. Soc. Pac. 125, 306 (2013).

[136] M. T. Busha, R. H. Wechsler, M. R. Becker, B. Erickson, and A.E. Evrard, in American Astronomical Society Meeting Abstracts (2013), Vol. 221, p. 341.07, https:// files.aas.org/aas221/AAS_221_Abstracts.pdf.

[137] P. Fosalba, E. Gaztañaga, F. J. Castander, and M. Crocce, Mon. Not. R. Astron. Soc. 447, 1319 (2015).

[138] H. Jeffreys, Theory of Probability, 3rd ed. (Oxford University, New York, 1961).

[139] P. Marshall, N. Rajguru, and A. Slosar, Phys. Rev. D 73, 067302 (2006).

[140] S. Singh, R. Mandelbaum, and S. More, Mon. Not. R. Astron. Soc. 450, 2195 (2015).
[141] C. L. Bennett et al. (WMAP Collaboration), Astrophys. J. Suppl. Ser. 208, 20 (2013).

[142] J. W. Henning et al., Astrophys. J. 852, 97 (2018).

[143] F. Beutler, C. Blake, M. Colless, D. H. Jones, L. StaveleySmith, L. Campbell, Q. Parker, W. Saunders, and F. Watson, Mon. Not. R. Astron. Soc. 416, 3017 (2011).

[144] A. J. Ross, L. Samushia, C. Howlett, W. J. Percival, A. Burden, and M. Manera, Mon. Not. R. Astron. Soc. 449, 835 (2015).

[145] M. Betoule et al. (SDSS Collaboration), Astron. Astrophys. 568, A22 (2014).

[146] J. L. Bernal, L. Verde, and A. G. Riess, J. Cosmol. Astropart. Phys. 10 (2016) 019.

[147] F. Kohlinger et al., Mon. Not. R. Astron. Soc. 471, 4412 (2017).

[148] L. Fu et al., Mon. Not. R. Astron. Soc. 441, 2725 (2014).

[149] C. Yeche, N. Palanque-Delabrouille, J. Baur, and H. du Mas des Bourboux, J. Cosmol. Astropart. Phys. 06 (2017) 047.

[150] Y. Fukuda et al., Phys. Rev. Lett. 81, 1562 (1998).

[151] S. Hinton, J. Open Source Software 1, 45 (2016). 